\title{
The SUMER spectral atlas of solar-disk features ${ }^{\star}$
}

\author{
W. Curdt ${ }^{1}$, P. Brekke ${ }^{2,3}$, U. Feldman ${ }^{4}$, K. Wilhelm ${ }^{1}$, B. N. Dwivedi ${ }^{1,5}$, U. Schühle ${ }^{1}$, and P. Lemaire ${ }^{6}$ \\ 1 Max-Planck-Institut für Aeronomie, 37191 Katlenburg-Lindau, Germany \\ 2 Institute of Theoretical Astrophysics, University of Oslo, Blindern, 0315 Oslo 3, Norway \\ 3 ESA Space Science Department / NASA Goddard Space Flight Center, Greenbelt, Md. 20771, USA \\ 4 E. O. Hulburt Center for Space Research, Naval Research Laboratory, Washington D.C., 20375, USA \\ ${ }^{5}$ Department of Applied Physics, Banaras Hindu University, Varanasi 221005, India \\ ${ }^{6}$ Institut d'Astrophysique Spatiale, Unité Mixte CNRS - Université de Paris XI, 91405 Orsay Cedex, France
}

Received 15 December 2000 / Accepted 7 March 2001

\begin{abstract}
A far-ultraviolet and extreme-ultraviolet (FUV, EUV) spectral atlas of the Sun between $670 \AA$ and $1609 \AA$ in the first order of diffraction has been derived from observations obtained with the SUMER (Solar Ultraviolet Measurements of Emitted Radiation) spectrograph on the spacecraft SOHO (Solar and Heliospheric Observatory). The atlas contains spectra of the average quiet Sun, a coronal hole and a sunspot on the disk. Different physical parameters prevalent in the bright network $(\mathrm{BN})$ and in the cell interior $(\mathrm{CI})$ - contributing in a distinct manner to the average quiet-Sun emission - have their imprint on the BN/CI ratio, which is also shown for almost the entire spectral range. With a few exceptions, all major lines are given with their identifications and wavelengths. Lines that appear in second order are superimposed on the first order spectra. These lines are clearly marked in the atlas. The spectra include emissions from atoms and ions in the temperature range $6 \times 10^{3} \mathrm{~K}$ to $2 \times 10^{6}$ K, i.e., continua and emission lines emitted from the lower chromosphere to the corona. This spectral atlas, with its broad wavelength coverage, provides a rich source of new diagnostic tools to study the physical parameters in the chromosphere, the transition region and the corona. In particular, the wavelength range below $1100 \AA$ as observed by SUMER represents a significant improvement over the spectra produced in the past. In view of the manifold appearance and temporal variation of the solar atmosphere, it is obvious that our atlas can only be a - hopefully typical - snapshot. Brief descriptions of the data reduction and calibration procedures are given. The spectral radiances are determined with a relative uncertainty of 0.15 to $0.30(1 \sigma)$ and the wavelength scale is accurate to typically $10 \mathrm{~m} \AA$. The atlas is also available in a machine readable form.
\end{abstract}

Key words. Sun: UV radiation - Sun: chromosphere - Sun: transition region - Sun: corona line: identification - atlases

\section{Introduction}

The EUV portion of the electromagnetic spectrum extends from $100 \AA$ to $1000 \AA$, whereas the wavelength range from $1000 \AA$ to $2000 \AA$ is commonly referred to as FUV portion in the literature. The solar spectrum in these ranges contains not only a large number of bright emission lines, mainly from the chromospheric and lower transition region plasmas, i.e., formed at electron temperatures $T_{\mathrm{e}} \leq 2.5 \times 10^{5} \mathrm{~K}$, but also many coronal lines formed at temperatures of one million kelvin and above. However, above $1216 \AA$, no allowed lines from the upper transition region have previously been observed. Only a few forbidden lines that originate at coronal temper-

Send offprint requests to: W. Curdt, e-mail: curdt@linmpi.mpg.de

* Table A.1 is only available in electronic form at the CDS via anonymous ftp to cdsarc.u-strasbg.fr (130.79.128.5) or via

http://cdsweb.u-strasbg.fr/cgi-bin/qcat?J/A+A/375/591 atures in plages had been reported in limb spectra (e.g., Brueckner 1981). In comparison, the solar spectrum in the range from $660 \AA$ to $1200 \AA$ includes a large number of lines from the upper transition region and corona, in addition to lines from the chromosphere and lower transition region (Curdt et al. 1997).

In this paper we present a spectral atlas representative of various solar features observed with SUMER on SOHO. SUMER is a high-resolution telescope and spectrograph designed to obtain stigmatic slit images with spatial and spectral resolution elements of $\approx 1^{\prime \prime}$ and $\approx 40 \mathrm{~m} \AA$ (in first order) as well as high temporal resolution over the wavelength range from $465 \AA$ to $1610 \AA$. The accessible range depends on which of the detectors is used. While detector "A" can in principle record spectra from $780 \AA$ to $1610 \AA$ in first order of diffraction, the range of detector "B" reaches from $660 \AA$ to $1500 \AA$. The gradual lower wavelength limit (for lines observed in second order) results from the steep fall-off of the reflectivity of the silicon carbide optics below $500 \AA$. The Ne VII line at 
$465 \AA$ represents the shortest wavelength identified so far with this instrument. The range from $660 \AA$ to $805 \AA$ can be covered in both orders. More than 1100 emission lines are available in the SUMER spectral range. These include resonance lines as well as previously unobserved faint intersystem lines, which can be detected by SUMER because of its more efficient low-noise detectors compared to previous instruments, as discussed by Feldman et al. (1997) and Curdt et al. (1997). Thus, the SUMER spectral atlas provides a rich source of new diagnostic tools for probing essential physical properties of the emitting plasma and studying electron densities, electron temperatures and elemental abundances throughout the solar atmosphere.

Earlier EUV/FUV high-resolution instruments made use of UV-sensitive photographic plates (or film) as detectors. Because of the fairly low efficiency of the photographic plates used, few (if any) spectra were obtained from regions that extended more than $20^{\prime \prime}$ above the limb. In comparison, SUMER has recorded line intensities extending out to $600^{\prime \prime}$ (Feldman et al. 1999). Off-limb features are, however, excluded here and will be covered by a separate communication.

Given the radiometric calibration of SUMER before and during the mission (Hollandt et al. 1996; Wilhelm et al. 1997a; Schühle et al. 1998, 2000), we are able to present the absolute spectral radiances of different solar features in the wavelength range from $660 \AA$ to $1485 \AA$ in the first order of diffraction using the detector "B". For the sake of completeness, we have added the spectral range from $1485 \AA$ to $1609 \AA$ taken from a "A"detector spectrum of the quiet Sun. The calibrated radiance spectra have been compared to well-calibrated full disk irradiance measurements and reasonable agreement is found. (Dammasch et al. 1999a). The absolute value of the radiation in the $\mathrm{EUV} / \mathrm{FUV}$ region is important for studying not only the solar transition region and corona, but also the atmosphere of the Earth. This radiation is an important source of energy in the upper atmosphere of the Earth and changes the temperature, chemistry and dynamics in these layers.

In Sect. 1, a brief summary is given of the most important EUV/FUV instruments and previously published solar spectral catalogues and line lists. In Sect. 2 we describe the instrument and the observation, while Sect. 3 deals with data reduction and calibration. The actual atlas is presented in Sect. 4. A list of all observed lines is added as annex to this atlas.

\section{Earlier spectral atlases}

Space astronomy started in 1946 in the United States when W. von Braun's A4 rocket engines (during the war named V-2 missile) became available to scientists for launching free-flying, high-altitude observatories. It was not surprising, under these circumstances, that a group from the US Naval Research Laboratory (NRL) became the first to observe the ultraviolet radiation of the Sun with a spectrograph mounted on the tail fin of a A4 rocket. Since then, much progress has been made in solar high-resolution spectroscopy from space (see Feldman et al. 1988). A large number of experiments have been launched to observe the EUV and FUV portions of the solar spectrum. High spectral and spatial resolutions were achieved by several instruments at wavelengths above $1150 \AA$. At shorter wavelengths, however, the spectral and spatial resolution were moderate and only strong lines could be observed and/or identified. Spectral catalogues and line lists covering the FUV and EUV portions of the spectrum have previously been published by Burton et al. $(1967)^{1}$, Burton \& Ridgeley $(1970)^{1}$, Dupree \& Reeves (1971), Gabriel et al. $(1971)^{1}$, Huber et al. $(1973)^{1}$, Malinovsky \& Heroux (1973), Kjeldseth-Moe et al. (1976), Doschek et al. $(1976)^{1}$, Feldman et al. $(1976 \mathrm{a}, 1976 \mathrm{~b})^{1}$, Vernazza \& Reeves (1978), Dere (1978) ${ }^{1}$, Feldman \& Doschek (1978), Cohen et al. $(1978)^{1}$, Cohen (1981), Sandlin et al. $(1986)^{1}$, Brekke et al. (1991), Feldman \& Doschek $(1991)^{1}$, Brekke (1993), Thomas \& Neupert (1994), Brooks et al. (1999).

In particular, two instruments made a significant impact on solar spectroscopy: The NRL/S082B EUV spectrograph on the Skylab ATM (Apollo Telescope Mount) and the NRL/High Resolution Telescope and Spectrograph (HRTS). The S082B instrument operated in the $970 \AA$ to $3940 \AA$ range, but due to the coating $\left(\mathrm{Al}+\mathrm{MgF}_{2}\right)$ of the optical surfaces, the instrument was very inefficient below $1100 \AA$ (Bartoe et al. 1977). A spectral atlas covering the $1175 \AA$ to $1950 \AA$ wavelength range based on the $\mathrm{S} 082 \mathrm{~B}$ spectrometer was published by Cohen (1981). The $2^{\prime \prime} \times 60^{\prime \prime}$ slit defined the spatial resolution element. Line lists based on the S082B data include those of Doschek et al. (1976), Feldman et al. (1976a, 1976b), Feldman \& Doschek (1978), and Cohen et al. (1978), the latter being the most comprehensive. A few very long exposures of bright solar features resulted in useful spectra at wavelengths as short as $970 \AA$. A line list compiled from these observations was published by Feldman \& Doschek (1991).

During the Skylab mission, several calibration rocket (CALROC) flights were carried out as part of a programme for calibration of the S082B spectrograph. A FUV spectral atlas in the wavelength range from $1170 \AA$ to $2100 \AA$ was prepared by Kjeldseth-Moe et al. (1976). The CALROC atlas contains the absolute intensity of an averaged quiet region located $300^{\prime \prime}$ inside the solar limb. During the observations the slit was rastered across its linear extension, thus averaging over an area of about $60^{\prime \prime} \times$ $60^{\prime \prime}$ on the solar disk. In addition the atlas contains a quiet region $50^{\prime \prime}$ inside the solar limb while an active region is presented for wavelengths above $1680 \AA$ A. During these observations, the full spatial resolution of the instrument was used. The spectral resolution of the data is $\approx 70 \mathrm{~m} \AA$ and the radiance measurements have estimated relative uncertainties of $\pm 25 \%$ ( $1 \sigma$ ) (Kjeldseth-Moe et al. 1976).

\footnotetext{
${ }^{1}$ References contain line lists with line identification, line strengths, etc., but no detailed spectral scans.
} 
This atlas was used as a reference calibration source for other FUV spectra for many years.

HRTS, which operated at wavelengths longer than $1150 \AA$, was the first spectrometer to combine both high spectral resolution $(50 \mathrm{~m} \AA)$ and good angular resolution $\left(1^{\prime \prime}\right)$ with extensive wavelength and spatial coverage (Bartoe \& Brueckner 1975). It was launched several times on sounding rockets and was part of the 5 day Spacelab 2 mission. Spectral catalogues based on the HRTS material covering the wavelength range from $1190 \AA$ to $1710 \AA$ have been published by Brekke et al. (1991) and Brekke (1993). These atlases represented different solar features that intercepted the $950^{\prime \prime}$ long slit extending from disk centre to the limb. The features included quiet Sun, plages, a sunspot (including a lightbridge), and an explosive event. A very comprehensive line list based on the HRTS material (and to some extent on the Skylab data) was published by Sandlin et al. (1986). The HRTS instrument has provided very important information on the properties of the solar chromosphere and transition region. Excellent reviews of some of the HRTS results can be found in the book "The Solar Transition Region" (Mariska 1992), and in Cook \& Brueckner (1979).

The LASP (Laboratory of Atmospheric and Space Physics) EUV spectrometer has been flown on sounding rockets (Hassler et al. 1991). This instrument has been the only solar instrument with an onboard wavelength calibration lamp to provide an absolute wavelength reference with high spectral resolution. During the first flight the wavelength range was $1520 \AA$ to $1600 \AA(760 \AA$ to $800 \AA$ in second order). During a later rocket flight on March 12, 1998 the spectrometer was tuned to cover the spectral ranges from $1200 \AA$ to $1280 \AA$ in the first order and $600 \AA$ to $640 \AA$ in the second order.

Before the SOHO mission, the wavelength region between $660 \AA$ and $1175 \AA$ remained poorly observed. Spectra covering the $300 \AA$ to $2950 \AA$ wavelength range with a spectral resolution of about $0.4 \AA$ were obtained during a series of rocket flights in the late 1960s and early 1970s by Burton et al. (1967), Burton \& Ridgeley (1970), and Gabriel et al. (1971). Due to low spectral resolution, line shapes could not be obtained. However, many intense solar lines were identified. Improved observations of the EUV solar spectrum in the range from $280 \AA$ to $1340 \AA$ were obtained with the Harvard College Observatory (HCO) spectroheliometer S055 on Skylab (Reeves et al. 1977) and smaller instruments flown earlier on Orbiting Solar Observatories OSO-4 and OSO-6 (cf. Huber et al. 1973).

The S082A on Skylab was a slitless objective-type grating spectrograph covering the wavelength range from $170 \AA$ to $630 \AA$ (Tousey et al. 1977). Each exposure included numerous images of the entire solar disk with a spatial resolution of $2^{\prime \prime} \times 2^{\prime \prime}$. An atlas of EUV spectroheliograms from $170 \AA$ to $625 \AA$ has been made available by Feldman et al. (1987).

Spectral catalogues at shorter wavelengths are those of Vernazza \& Reeves (1978) and Malinovsky \& Heroux
(1973). Based on observations with the S055 spectrometer on Skylab, Vernazza \& Reeves (1978) presented a spectral atlas of different solar features in the wavelength range from $280 \AA$ to $1350 \AA$ with a spectral resolution of $1.6 \AA$. Until now this atlas represented the most complete set of quiet-Sun spectra in this wavelength range. But due to poor spectral resolution of this spectrometer, many lines reported in this atlas are incorrectly identified. More recently an active region EUV atlas in the range from $230 \AA$ to $450 \AA$ was obtained with the SERTS (Solar EUV Rocket Telescope and Spectrometer) rocket experiment (Thomas \& Neupert 1994). Emission lines of molecular hydrogen, which are present in sunspot spectra, have been measured and/or predicted by Bartoe et al. (1979). Brooks et al. (1999) have presented a spectral line list in the ranges $308 \AA$ to $381 \AA$ and $513 \AA$ to $633 \AA$ based on measurements of the normal incidence channel (NIS) of the SOHO/CDS instrument (Coronal Diagnostic Spectrometer, Harrison et al. 1995). A calibrated solar EUV spectrum in the same wavelength range has also been presented by Brekke et al. (2000).

\section{Instrumentation and observations}

SUMER is part of the SOHO mission of ESA and NASA. SOHO was launched on December 2, 1995 by an Atlas II-AS Centaur into a transfer trajectory to the first Lagrangian point, L1. It was injected into a halo orbit around L1 on February 14, 1996 where, in continuous view of the Sun, it accompanies the Earth at a sunward distance of $1.5 \times 10^{6} \mathrm{~km}$. SOHO lost its attitude control on June 25, 1998, but was subsequently recovered later in 1998 .

This section describes details of the instrument and the data acquisition which are relevant for the interpretation of the data. A comprehensive description of the instrument is given by Wilhelm et al. (1995), and first results and inflight performance characteristics are given by Wilhelm et al. (1997b) and Lemaire et al. (1997).

\subsection{Instrument}

SUMER is a stigmatic normal-incidence spectrograph operating in the range from $465 \AA$ to $1610 \AA$ with optical elements made of silicon carbide ( $\mathrm{SiC})$ and three normalincidence reflections. Only a few lines below $500 \AA$ have so far been observed with SUMER. The off-axis parabola telescope mirror has a plate scale in the slit plane of $6.3 \mu \mathrm{m} /$ arcsec. It can be moved to obtain disk and offlimb spectra in the lower corona. Four slits with angular dimensions of $4^{\prime \prime} \times 300^{\prime \prime}, 1^{\prime \prime} \times 300^{\prime \prime}, 1^{\prime \prime} \times 120^{\prime \prime}$, and $0.3^{\prime \prime} \times 120^{\prime \prime}$ are available, the two short slits can be placed at three different spatial positions with respect to the detector. We have used the $1^{\prime \prime} \times 300^{\prime \prime}$ slit (slit \#2) during the coronal-hole and quiet-Sun observations given in this atlas. The sunspot was observed using the narrow $0.3^{\prime \prime} \times$ $120^{\prime \prime}$ slit (slit \#7).

Two diffraction orders can be observed by SUMER; first order lines and second order lines appear superimposed in the spectrum. A few lines could also be observed 
in third order (Feldman et al. 1997). The dispersion of the instrument is slightly wavelength dependent. For detector "B", it varies from $44.7 \mathrm{m \AA} /$ pixel (first order) and $22.3 \mathrm{~m} \AA /$ pixel (second order) at $660 \AA$ to $41.2 \mathrm{~m} \AA /$ pixel and $20.6 \mathrm{~m} \AA /$ pixel at $1500 \AA$.

The instrument is equipped with two photoncounting detectors ("A" and "B") with image encoding in cross-delay-line technique (XDL), for details see Siegmund et al. (1994). Only one detector can be operated at a time. Each detector has 1024 spectral and 360 spatial pixels. The pixel size of approximately $26.5 \mu \mathrm{m} \times 26.5 \mu \mathrm{m}$ is defined by the analogue electronics. The plate scale in the focal plane of the spectrometer is $\approx 1$ pixel/arcsec resulting in an effective focal length of the instrument of $5.5 \mathrm{~m}$. By centroiding, wavelength measurements can be performed with a precision of $5 \mathrm{~m} \AA$ and better if sufficient lines are available for this purpose (cf., Dammasch et al. 1999b). The central area of the detector is coated with $\mathrm{KBr}$ (potassium bromide). This coating increases the detection quantum efficiency (DQE) mainly in the range from $900 \AA$ to $1500 \AA$. Figure 1 shows a detector readout as raw data in the spectral range from $746 \AA$ to $791 \AA$ in the bottom panel. Observations of lines on both sections of the photocathode can be used to discriminate second order lines from first order lines, since the photocathode responsitivity changes differently for lines in different orders. Approximately 50 spectral pixels at the extreme ends of the detectors are covered by a mesh providing a 1:10 attentuation for $\mathrm{H}$ I Ly $\alpha$ observations.

During the radiometric laboratory calibration (Hollandt et al. 1996), the responsivity of SUMER was determined for both detectors with a transfer standard light source. In-flight calibration refinements indicate that this calibration was valid and stable until the SOHO accident in June 1998. The dark signal of the detectors is extremely low, and for disk observation, scattered light can be neglected. Both detectors show non-uniformity effects typical for micro-channel-plate (MCP) intensifiers. These effects stem from the MCP structure, the inhomogeneity of the electric field, electronic non-linearities, and individual pixel deficiencies. These effects, which are very worrisome for the purpose of imaging, can be compensated to some extent by flat-field and geometric corrections. SUMER regularly generates a flat-field matrix, which can be applied either on board or on the ground.

\subsection{Data acquisition}

The standard observing sequence for the "reference spectrum" consists of a series of full detector readouts at different wavelengths. To cover the wavelength range of detector "B" from $670 \AA$ to $1500 \AA$, a set of 61 spectral sections, each offset by $\approx 12.8 \AA$ (in first order), were obtained sequentially. This offset was used to record the entire spectral range on both the bare and the $\mathrm{KBr}$-coated part of the MCP, which allows us to discriminate second order lines from first order lines. For this exercise, it was extremely helpful that the range from $670 \AA$ to $750 \AA$ was recorded in both orders on this detector. The spectral catalogues, presented here, have been extracted from the KBr-coated portion of the detector, except for the wavelength region around a telemetry gap (see below) and around H I Ly $\alpha$, where only the bare part is available. Note that we have to place this strong line on the attenuator section located at the extreme pixel positions of the detector. Also, the extreme sections of our spectral range can only be recorded on the bare part of the detector. The sunspot spectrum was obtained using a modified observing sequence for faster data acquisition, which was composed of 37 spectral windows, displaced by larger increments of $\approx 20.8 \AA$ each.

Whenever possible, the reference spectrum was preceded by a raster sequence where the spectrometer slit moves perpendicular to the slit direction to map an area of $120^{\prime \prime}$ width. This raster contains 157 slit positions with a step size of $0.75^{\prime \prime}$. With an exposure time of $10 \mathrm{~s}$ at each spatial position the complete raster scan takes 26 min. Three spectral windows centred on selected emission lines were extracted from the detector at each slit position. Monochromatic images at different wavelengths can be constructed from the raster sequence by summing up the counts in the line profiles at each spatial location. Images of the Extreme-Ultraviolet Imaging Telescope (EIT) onboard SOHO (Delaboudinière et al. 1995) were also taken as context images and used to find the exact slit position during the observation as well as to co-align the SUMER observations with those obtained with other instruments. After the raster sequence, the pointing returns to the target position at the centre of the raster image where the reference spectrum is to be acquired. This solar position is maintained if the solar-rotation tracker is activated.

About 300 SUMER reference spectra have been recorded from different locations of the Sun so far. We have selected the reference spectrum obtained on April 20, 1997 as a good choice for the quiet Sun, because neither eruptive phenomena are found in this data set nor do telemetry gaps deteriorate the data quality. From $1485 \AA$ onwards, we have added quiet-Sun "A"-detector spectra taken on August 12, 1996 in order to present the entire SUMER spectrum of the quiet Sun up to $1609 \AA$.

The criteria for typical coronal-hole spectra provide grounds for debate. There seems to be a gradual scale of the strengths of coronal holes. While our spectrum, recorded near disk centre on October 12, 1996, is characterized by a depression of approximately a factor of two through the entire wavelength range, this difference is only found at wavelengths below $1000 \AA$ in a polar coronal-hole spectrum (cf. Schühle et al. 1999). This aspect is not yet understood and needs further investigation.

We have selected the sunspot spectrum obtained on March 18, 1999 for our atlas, because this spot was one of the largest observed by SUMER so far and also because the slit seems to have been placed right through the central umbral part. However, we lost a small portion of this spectrum $(2 \AA)$ during a telemetry gap. A spectroscopic 


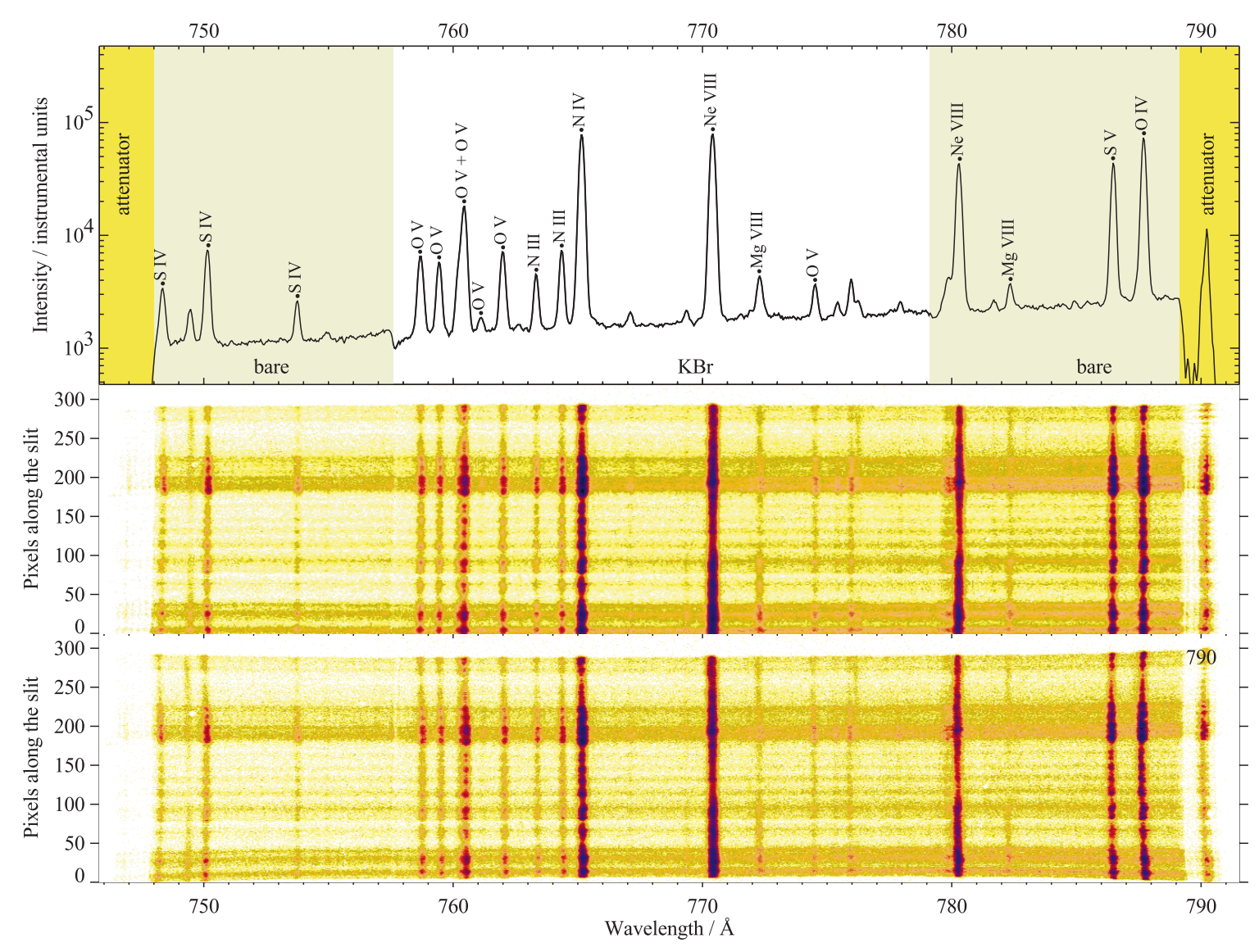

Fig. 1. The SUMER spectral window centred around the Ne VIII $770 \AA$ line, with an exposure time of 300 s, obtained near disk centre of the quiet Sun. The entire detector readout is shown in the lower panel as raw data and after flat-field correction and geometrical distortion correction in the central panel. The spectral profile in the upper panel, integrated from spatial pixels 35 to 174 , provides some line identifications. The changes in the continuum counts at $758 \AA$ and $780 \AA$, which are caused by the different responsivity of the $\mathrm{KBr}$ photocathode compared to the bare $\mathrm{MCP}$, are much more prominent at longer wavelengths.

analysis of this spectrum has been published in more detail by Curdt et al. (2000a). Context images showing the position of the slit during data acquisition are presented in Fig. 2. All observational parameters are summarized in Table 1.

\section{Data reduction}

The spectra presented here have been recorded with detector "B" and compressed during downlink. After decompression, the data set was flat-field corrected and the geometric distortion of the detector removed. Finally, spectral pixels were converted to wavelengths and the intensity given in physical units. Standard procedures have been applied for the basic data processing.

\subsection{Correction for flat-field and geometric distortions}

As mentioned in Sect. 3.1, the responsivity of the detector is nonuniform on scales of about 20 pixels or less. Several flat-field matrices have been acquired by long exposures of three hours in the H I Lyman continuum at $880 \AA$ while the spectrometer was defocussed. This provides a deep, al- though not entirely uniform exposure which the SUMER processor compares against a median-20 filtered array in order to extract all non-uniformities smaller than 20 pixels. The small-scale variation amounts to as much as $50 \%$. This includes a pixel-to-pixel variation of approximately $20 \%$ in the spatial dimension caused by an analogue-todigital converter differential non-linearity.

The properties of the detector vary as a function of the extracted charge as the detector is being "scrubbed" by the accumulated counts. Thus, the flat-field data need to be updated quite frequently. In all cases, we selected the latest flat-field image obtained before the considered observation for this process.

The fringe fields in the detector MCP-anode gap lead to a geometric distortion which makes the image of the slit shorter at the centre of the detector compared to areas closer to the edges. As a result, the spectral lines are curved (cf. Fig. 1). An artificial "rectangular grid" has been produced using averaged stable solar spectral lines and continuum data, from which the distortion has been parametrized. This correction is then applied to the data in order to overcome this shortcoming. The SUMER standard "destretching procedure" (Moran 1996) also contains a compensation for the small inclination of the slit image 


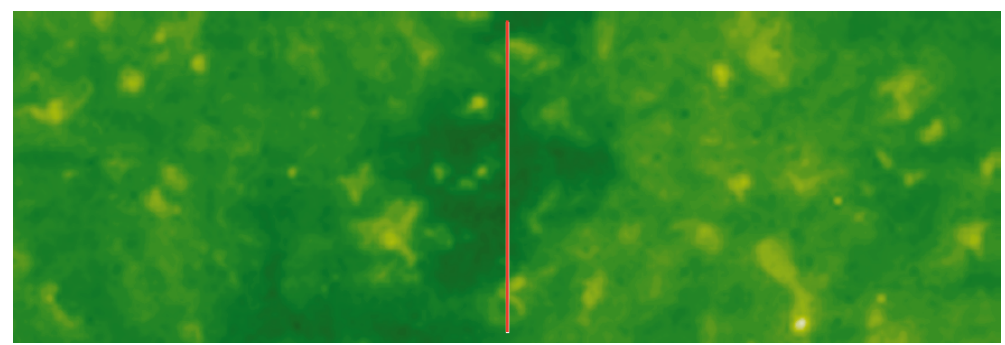

EIT $195 \AA$

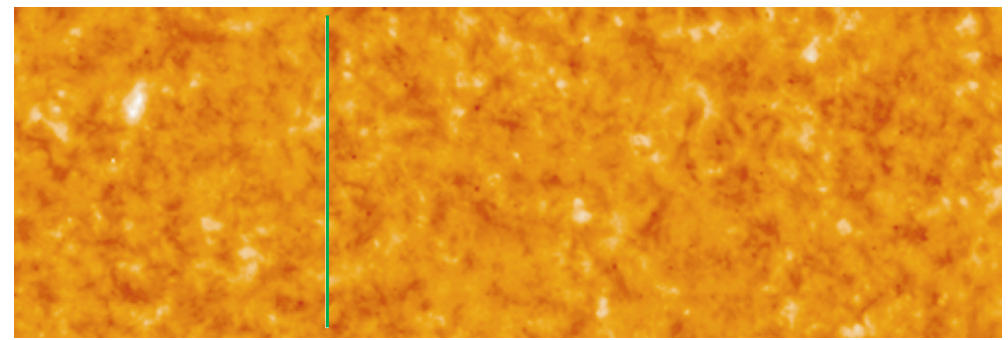

EIT $304 \AA$

Quiet Sun 20-Apr-97

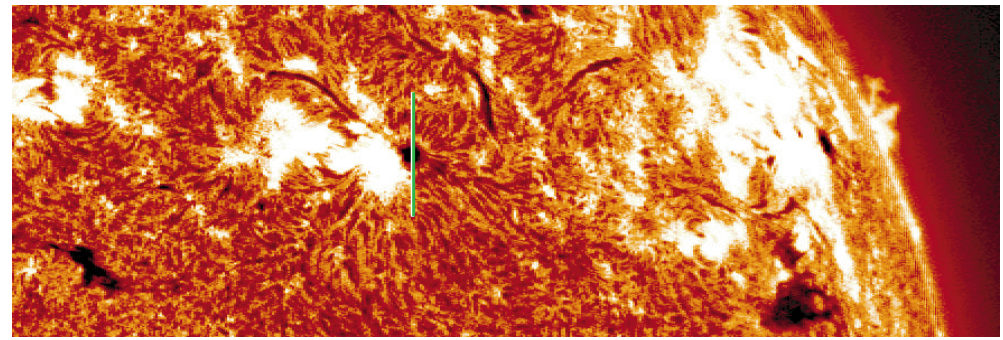

BBSO $6565 \AA$

Sunspot 18-Mar-99

Fig. 2. Context images showing the position of the SUMER slit during data acquisition. The field of view is $937^{\prime \prime} \times 320^{\prime \prime}$ in all three cases. Top: The equatorial coronal hole seen as a dark structure in the FexiI/195 $\AA$ channel (also seen in other EIT channels, courtesy: EIT consortium). Centre: A quiet-Sun area near disk centre in the He II/304 $\AA$ channel (courtesy EIT consortium). Bottom: The leading sunspot of active region NOAA 8487 as seen on a H i H $\alpha$ filtergram (courtesy: Big Bear Solar Observatory).

Table 1. Observational parameters of the data sets presented in this atlas. The pointing in $x$ and $y$ is given in seconds of arc (SOHO co-ordinates) and refers to slit centre at the start of the observation, $t_{0} . T$ is the exposure time in seconds and $d$ is the total duration of the spectral scan in hours. $n_{\mathrm{p} x}$ gives the number of pixels which have been used for averaging along the slit. The solar rotation tracking was active in all cases.

\begin{tabular}{|c|c|c|c|c|c|c|c|c|c|c|}
\hline target & detector & date & $\begin{array}{c}t_{0} \\
\mathrm{UT}\end{array}$ & $\begin{array}{l}d \\
\mathrm{~h}\end{array}$ & $\begin{array}{l}x \\
\prime \prime\end{array}$ & $\begin{array}{l}y \\
\prime \prime\end{array}$ & $\begin{array}{l}T \\
\mathrm{~s}\end{array}$ & $\begin{array}{c}n_{\mathrm{p} x} \\
\text { pixel }\end{array}$ & $\begin{array}{c}\text { slit } \\
\#\end{array}$ & rotcomp \\
\hline coronal hole & B & October 12,1996 & $20: 45$ & $5: 15$ & -66 & -26 & 300 & 171 & 2 & on \\
\hline quiet Sun & B & April 20, 1997 & 00:03 & $5: 09$ & 0 & 0 & 300 & 300 & 2 & on \\
\hline sunspot & B & March 18, 1999 & $17: 36$ & $2: 51$ & 468 & 360 & 90 & 17 & 7 & on \\
\hline quiet Sun & $\mathrm{A}$ & August 12, 1996 & 01:13 & $2: 07$ & 0 & 0 & 113 & 115 & 4 & on \\
\hline
\end{tabular}

relative to the detector pixel direction, which is caused by a residual alignment error between detector anode and spectrometer grating.

Figure 1 shows a full-detector image centred at $\approx 769 \AA$ before and after the corrections for flat-field and geometric distortions are made. As seen in the central panel, the continuum features appear to be horizontal in the dispersion direction and the emission lines are straight to within one spectral pixel after this correction.

\subsection{Wavelength calibration}

Since there is no absolute wavelength reference available in the spectrometer, a wavelength scale can only be derived using solar chromospheric lines. The dispersion changes as a function of wavelength, and so each exposure needs an individual calibration. The wavelength calibration is based on identifying the position of chromospheric lines on the detector and the assumption of neglibible net Doppler flows for these lines. In quiet regions atomic lines and lines from singly ionized species are formed over a limited range in temperature and other physical conditions, and are known to show relatively small average absolute shifts (e.g., Samain 1991). Also, the observed small velocity variations along the slit in these lines indicate that these lines are very useful for establishing an absolute wavelength scale.

The pixel-to-wavelength relation is achieved by a correlation of the line centroids in the entire $43 \AA$ window with all known reference wavelengths in this window, 
preferably emission lines from neutrals and singly ionized species, which are fairly strong and unblended in the solar spectrum and for which the absolute wavelengths are known with high accuracy. Since the non-linear dispersion is known very accurately from the optical design, this correlation leads to a constant offset for each exposure. In an iterative process, we inspected the preliminary wavelength calibration of each individual exposure for inconsistencies and deviations. This exercise has been very useful in eliminating misidentifications or finding problems with literature values for some of the reference lines.

The identification of reference lines is sometimes difficult due to the presence of many overlapping lines and also due to the presence of prominent lines both in first and second order in the SUMER spectrum. Except for a few close blends, the line centroids could be determined by multi-Gauss fits with estimated uncertainties of the order of 0.1 pixel $(\approx 5 \mathrm{~m} \AA$ in first order). The accuracy of the laboratory wavelengths of atomic lines is generally better than $2 \mathrm{~m} \AA$. Most of the laboratory wavelengths used for the wavelength calibration were taken from Kelly (1987).

After the spectra have been calibrated, the wavelengths should be accurate to typically $10 \mathrm{~m} \AA$ or 2 to $5 \mathrm{~km} \mathrm{~s}^{-1}$ on a velocity scale which is relative to quietSun chromospheric layers. We do not claim this accuracy for the sunspot spectra, where this value can easily reach $40 \mathrm{~m} \AA$ in cases where either the measured line or the reference lines or both are shifted by net Doppler flows. It is also evident that the sunspot spectrum is more noisy, due to acumulative effect of the narrow slit, the shorter exposure time, and the reduced number of averaged pixels, which amounts to a factor of $\approx 100$ less counts.

In several cases, our measured wavelength values, which are reported in the annexed line list, suggest that the literature values have to be revised, in particular, this is the case for wavelengths of forbidden transitions in highly-ionized species, which are difficult to measure in the laboratory. Examples of more accurate measurements, which are beyond the scope of this atlas, are reported by Dammasch et al. (1999b) and Peter \& Judge (1999).

\subsection{Radiometric calibration}

The calibration of the spectral response of the SUMER instrument is based on a comparison with a radiometric transfer standard source, which had been calibrated against the Berlin Electron-Storage ring for SYnchrotron radiation (BESSY I) as primary radiometric standard (Hollandt et al. 1996). The transfer source provided 16 emission lines with known photon fluxes of rare gases in the SUMER wavelength range. These have been used as the basis for establishing the spectral responsitivity curves of the instrument in first and second order for both detectors.

Based on the experience with previous solar UV missions the stability of the calibration was a major concern. The combined effects of molecular organic contamination and solar irradiation was known to cause degradation of the optical performance in space. Therefore, a comprehensive cleanliness control programme was made part of the SUMER project, which successfully avoided this type of contamination. This could be verified by monitoring the sensitivity during the mission (Schühle et al. 1998). The responsivity of the instrument has been monitored from the moment when first light was received on the telescope. The count rates for well known solar lines at quiet solar conditions were measured and found to be the same as predicted by the calibration made on the ground. During the entire mission, calibration measurements have been carried out at regular time intervals to track the responsivity of the instrument. The radiance at selected wavelengths of a quiet-Sun area was monitored to detect possible changes in the response. Despite the high variability of the Sun as a source, these measurements confirmed the stability of the calibration within uncertainty limits of $\pm 15 \%(1 \sigma)$ for detector "A" (Wilhelm et al. 1997a) and $\pm 20 \%$ for detector "B", in the range from $537 \AA$ to $1250 \AA$ (Schühle et al. 2000). In addition, the spectral calibration curves could be refined during flight by measuring solar line ratios and by observation of standard UV stars (Wilhelm et al. 1997a).

Thus we believe that in the wavelength range from $537 \AA$ to $1250 \AA$ the radiometric calibration has been valid until June 1998, the time when the loss of the SOHO attitude control occurred. Our spectrum of the sunspot was taken after recovery of the spacecraft when a change in sensitivity of the SUMER instrument was discovered. Data after the recovery are treated with a correction factor of $43 \%$ to account for the average loss of sensitivity attributable to the loss of SOHO. The uncertainty in the determination of this change affects also the overall uncertainty of the radiometric calibration after recovery which we estimate to $30 \%$. At wavelengths longer than $1250 \AA$ the uncertainties are $30 \%$ before and $40 \%$ after the SOHO recovery.

However, some corrections are necessary for bright lines. The count-rate capabilities of the detectors are slightly exceeded if bright lines are placed onto the $\mathrm{KBr}$ part of the photocathode, leading to a local-gain depression of the detector channel plates and dead-time effects of the electronics (Wilhelm et al. 2000). As a result the intensities of the lines affected (C III $977 \AA$, H I Ly $\beta 1026 \AA$, O vi $1032 \AA$, O vi $1037 \AA$, H i Ly $\alpha 1216 \AA$, O I 1302$1306 \AA$, C II 1335/1336 $\AA$ ) are underestimated in the raw data. The corrections, which amount to as much as $30 \%$ for the $\mathrm{C}$ III line and about $17 \%$ for the O VI, H I, O I, and $\mathrm{C}$ II lines have been applied in our atlas. The given intensity of the H I Ly $\alpha 1216 \AA$ line is only an approximation, since this bright line could only be observed with the attenuator. Since the brightness of structures observed on the Sun may have changed while the spectral range was covered, the intensities of lines may become less comparable with increasing difference in wavelength.

In the sunspot spectrum, the photon rate of some emission lines heavily exceeded the detector capabilities. 
Then, in addition to dead-time losses, some pulses are registered at a displaced position and appear in both dimensions as ghosts in an image. These electronic ghosts have been eliminated and appear as gaps in our spectrum. This correction was needed for H i Ly $\gamma 973 \AA$, C III $977 \AA$, O VI $1032 \AA$, O vi $1037 \AA$, H r Ly $\alpha 1216 \AA$, O v $1218 \AA \&$ Mg x $1219 \AA / 2, \mathrm{~N}_{\mathrm{V}} 1238 \AA$, and N v $1242 \AA$.

To confirm the consistency of the radiometric results further, we compared our calibration with other solar spectral instruments which measure the irradiance from the full solar disk. In using the spectral radiance of this atlas for a comparison with irradiance data from the full Sun, one has to take into account contributions from different features on the disk, such as active regions and coronal holes, and center-to-limb variation (limb brightening), which are not resolved in full-disk measurements. Full-disk irradiances have been reported for a number of selected emission lines for which full-Sun raster scans have been made with the SUMER instrument (Wilhelm et al. 1998). From these measurements the effects of active regions, coronal hole deficiencies, and detailed center-to-limb radiance variations have been determined. Most lines of the solar transition region are from optically thin plasmas and show substantial limb brightening, which leads to an average radiance of the solar disk of approximately twice the radiance at disk centre. Therefore, the irradiance from the full Sun will be higher than from the disk centre for most of the lines in this spectral range. Work has been done to compare the irradiances of the quiet Sun measured with SUMER with previous results in the literature, and whenever a comparison could be made, we found agreement within the uncertainty margins (Dammasch et al. 1999a). A comparison of the SUMER radiance spectrum has also been made with the irradiance spectrum of the SolarStellar Irradiance Comparison Experiment (SOLSTICE) on the Upper Atmospheric Research Satellite (above $1150 \AA$ ) and the EUV Grating Spectrograph (EGS) (below $1190 \AA$ ), which was flown on sounding rockets (Woods et al. 1998a). The latter two instruments have been calibrated against the Synchrotron Ultraviolet Radiation Facility (SURF) of the US National Institute of Standards and Technology (NIST) as primary radiometric standard. The comparison between the SOLSTICE/EGS irradiance spectrum and the SUMER quiet-Sun radiance spectrum was made between $800 \AA$ and $1600 \AA$ at a spectral resolution of $3 \AA$ (Schühle et al. 1998). For most parts of the spectrum the agreement was found to be very good, except where we have dominating secondorder lines in the SUMER spectrum and a spectral region around $950 \AA$, which could be attributed to an instrumental effect (Woods 1998b). A detailed comparison between SOLSTICE and SUMER in the range from $1200 \AA$ to $1560 \AA$ was given by Wilhelm et al. (1999). The comparison of stellar FUV spectra with the spatially resolved spectra of the Sun is also of great interest. Figure 3 compares the average quiet-Sun radiance spectrum in the range from $1287 \AA$ to $1307 \AA$ to the irradiance spectrum of the solar twin G2 V star $\alpha$ Cen A (for details, cf. Ayres 2000) as measured by the Hubble Space Telescope Imaging Spectrograph (HST-STIS). Both spectra are very similar, except for second order lines, which are not present in STIS spectra. Faint lines can be easier seen in the SUMER spectrum, which has a better photon statistic. The STIS spectrum with its better spectral resolution is very useful for studying line blends and line reversals due to optical thickness effects. A more detailed analysis and a radiometric comparison are beyond the scope of this atlas.

\section{Description of the atlas}

The spectra presented in this atlas are composites of individual exposures, most of them on the $\mathrm{KBr}$ photocathode. The separation wavelengths are indicated by broken vertical lines at the bottom. Although we have used the onboard solar rotation tracker for all spectra presented here, there are clear indications of evolution in the network structures observed. This has to be taken into account, if radiances of lines from different exposures are compared. The variability of the solar atmosphere on all temporal and spatial scales is amazing. These include periodic phenomena (cf., e.g., Curdt \& Heinzel 1998 and references therein) and explosive phenomena (cf., e.g., Innes et al. 1997 and references therein). To give an example, the emission measure of some lines increased by a factor of $\approx 5$ during the sunspot observation as derived from a comparison of lines recorded in both orders of diffraction (i.e., at the beginning of the sequence and at the end of the sequence).

The spectra are displayed as profiles in different colours, the quiet-Sun spectrum as black line, the coronalhole spectrum as blue line, and the sunspot spectrum as red line. In the sunspot profile the continuum background has been moderately noise-filtered. The BN/CI ratio of the quiet-Sun spectrum is overlayed as green, solid line using a separate axis scale. In Fig. 4 the spectrum is presented in portions of $42 \AA$ per page with $2 \AA$ of overlap. The atlas is also available in machine readable form via ftp or the internet ${ }^{2}$. This atlas represents our most up-to-date knowledge of the solar-disk spectrum in the given spectral range. Coronal and flare spectra have also been analyzed (Curdt et al. 2000b; Feldman et al. 2000), but are not the subject of this communication.

\subsection{Line identification}

Each resolved emission line is indicated by a mark, the measured wavelength in angstrom $(\AA)$, and the identification, if available. For the sake of a concise presentation, the wavelength values are restricted to three digits, one digit before and two rounded digits after the decimal point (e.g., the Ne VIII line observed at $770.423 \AA$ reads 0.42 in first order and 0.85 in second order). The mark can

\footnotetext{
2 Profiles and line list available via anonymous ftp to cdsarc.u-strasbg.fr (130.79.128.5) or via http://cdsweb.u-strasbg.fr/cgi-bin/qcat?J/A+A/375/591
} 


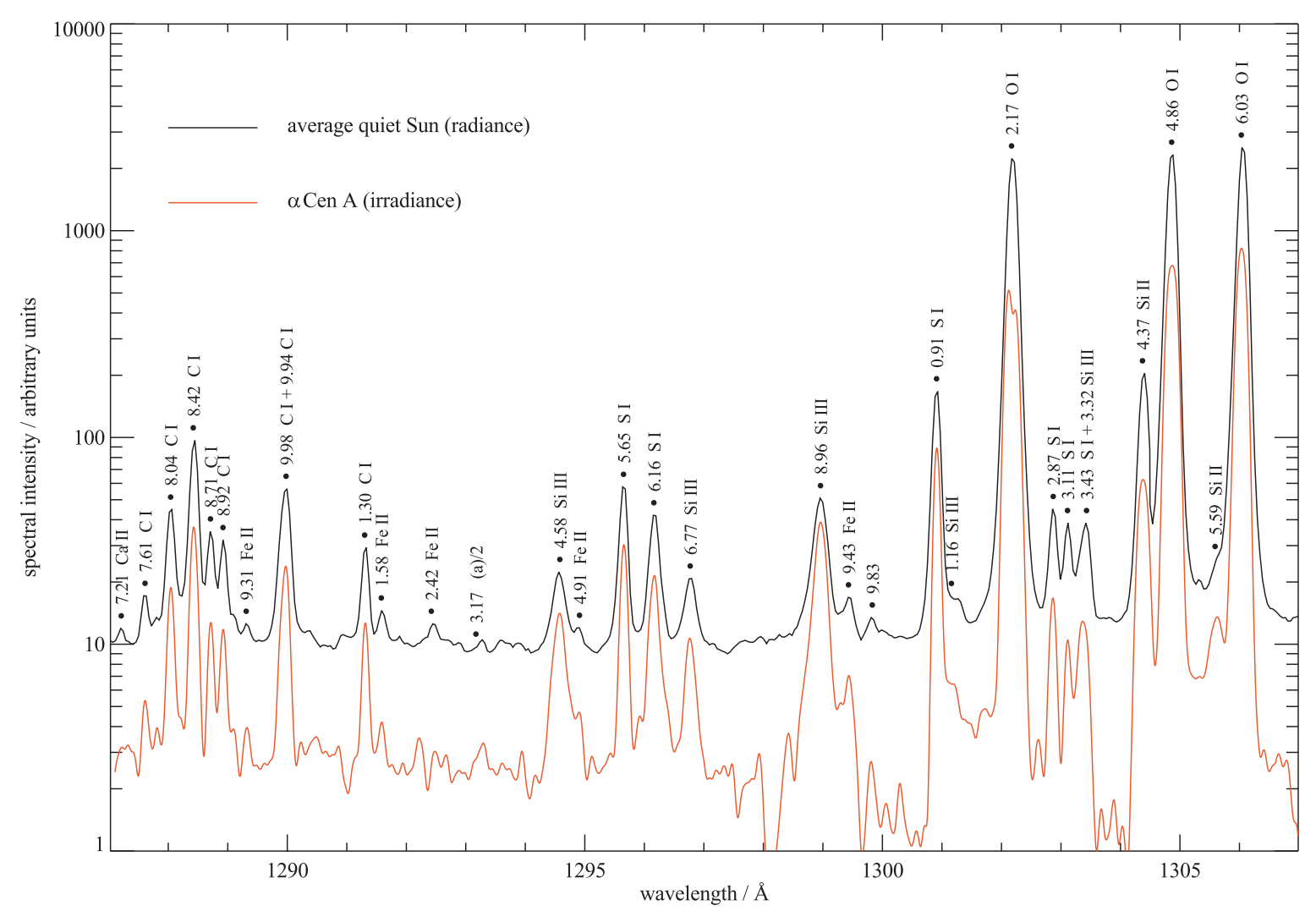

Fig. 3. Comparison of the FUV irradiance spectrum of $\alpha$-Cen A and of the quiet Sun radiance spectrum at disk centre in the range from $1270 \AA$ to $1310 \AA$. The stellar spectrum (courtesy: HST-STIS consortium) has been degraded to the SUMER resolution by a convolution with a Gaussian of standard deviation $\sigma=80 \mathrm{~m} \AA$.

be used as a cross-reference to line lists available in the literature, where additional spectroscopic information is found. Squares and circles point to line lists published on SUMER observations, circles to Curdt et al. (1997) and squares to Feldman et al. (1997), respectively. Diamonds point to the older lists of Kelly (1987), Cohen et al. (1978), Sandlin et al. (1986), and Bartoe et al. (1979). We have checked the line lists in this order and emphasize that marks are not meant in a sense of "first identification by", but, on the contrary, in general are pointing to the most recent list. Triangles denote lines observed for the first time or new identifications, these are mainly found in the sunspot spectrum. Open marks represent lines which are observed in second order of diffraction. Questionable identifications are marked by a question mark (?). Only $4 \%$ of the lines in the quiet-Sun spectrum remain unidentified at this stage, most of them being faint. This percentage increases to $12 \%$, if we also consider the sunspot spectrum, which seems to be dominated by 3 - to 6 -fold ionized species and is more difficult to interpret. In these cases we have given the temperature classification of Feldman et al. (1997), if available (cf. Fig. 4). A list of all lines observed in the SUMER spectral range is given electronically in the Annex.

\subsection{Bright network/cell interior ratio}

In the quiet-Sun spectrum, the SUMER slit cuts five partly sub-structured supergranular cells. We have sep- arated 21 pixels representative of bright network and 71 pixels of cell interior, respectively. The BN/CI ratio, thus derived from the same exposures, is $\geq 1$ at all wavelengths. It shows an interesting variation, depending on the particular emission line under consideration. This feature is a direct consequence of the plasma temperature and density of the emitting source. For lines with high formation temperature, the BN/CI ratios are not far from 1 , while they can reach values $>10$ for transition region lines.

\section{Summary}

The spatially resolved solar EUV/FUV spectra studied in this atlas, cover a wide spectral range from $670 \AA$ to $1609 \AA$ and provide a wealth of information on solar plasma structures from the upper chromosphere through the transition region to the corona. This information has, therefore, a tremendous diagnostic value for the emitting source. Together with the high spatial resolution of the SUMER spectrograph, compared to other solar EUV spectrometers flown during the last decades, plasma parameters of small solar features can be investigated. The atlas also provides an excellent reference for astrophysical applications. The SUMER spectrograph combines better spectral and spatial resolutions as well as coverage than any previous observations in the same wavelength range, and permits the extensive use of spectroscopic techniques in determining temperatures, pressures, densities and velocities in the upper solar atmosphere. The atlas also presents 
a powerful tool for the planning of future observations, i.e., to determine adequate integration times, to identify possible blends, and to select proper data extraction windows in upcoming solar studies.

Acknowledgements. We thank Tom Ayres for his help in retrieving the stellar data from the ST Science Institute archive.
The SUMER project is financially supported by DLR, CNES, NASA and the ESA PRODEX Programme (Swiss contribution). SUMER is part of SOHO, the Solar and Heliospheric Observatory, of ESA and NASA. U. F. acknowledges financial support from NRL/ONR 6.1 basic research programs and NASA grants.

\section{Appendix A: ${ }^{3}$}

Table A.1. Line List - List of spectral lines in the wavelength range from $668 \AA$ to $1611 \AA$ identified in SUMER spectra of the average quiet Sun (QS), a coronal hole $(\mathrm{CH})$ and a sunspot on disk (SS). Spectral lines observed in second order of diffraction which are also given here, extend the lower wavelength limit to below $500 \AA$. For each entry we give the observed wavelengths in angstrøm, the identification, the transition, the peak of spectral radiance, $L^{\text {peak }}$, in $\mathrm{mW}$ ( $\left.\mathrm{sr} \mathrm{m}^{2} \AA\right)^{-1}$ (incl. background), and a cross-reference to other line lists available in the literature (cf., Sect. 5.1). For second-order lines radiance entries are generally not provided, since the background separation in both orders of diffraction is a non-trivial task, which can not be automated. Only a few radiance values of strong second-order lines with negligible first-order contribution are given, which are marked by an asterisk $\left(^{*}\right)$. This line list complements Fig. 4 of the SUMER Spectral Atlas.

Fig.4. The SUMER solar-disk spectral atlas includes profiles of the average quiet Sun (black), an equatorial coronal hole (blue), and a sunspot (red). Resolved emission lines are indicated by a mark, the measured wavelength in angstrom ( $\AA$ ), and the identification, if available. The marks point to line lists available in the literature, where additional information about a specific line can be found. Squares ( $\square$ ) point to Feldman et al. (1997), circles (॰) to Curdt et al. (1997), diamonds ( $\diamond)$ to Kelly (1987), Cohen et al. (1978), or Sandlin et al. (1986). Triangles $(\triangle)$ are new lines or identifications. Filled symbols denote lines observed in first order of diffraction, open symbols are second-order lines. Only the three least-significant digits of the wavelength values are given. If available, unidentified lines are characterized by the temperature classification of Feldman et al. (1997) $\left(\mathrm{a}: T_{\mathrm{e}}<3 \times 10^{5} ; \mathrm{b}: T_{\mathrm{e}} \approx 3 \times 10^{5} ; \mathrm{c}: T_{\mathrm{e}} \approx 4 \times 10^{5} ; \mathrm{d}: 6 \times 10^{5}<T_{\mathrm{e}}<9 \times 10^{5} ; \mathrm{e:} T_{\mathrm{e}} \approx 1.4 \times 10^{6} ;\right.$ f: $T_{\mathrm{e}} \approx 1.8 \times 10^{6}$. The profiles have been radiometrically calibrated assuming first order of diffraction and the radiance axis is scaled to $\mathrm{mW} \mathrm{sr}^{-1} \mathrm{~m}^{-2} \AA^{-1}$. We have taken care of the type of photocathode (bare or $\mathrm{KBr}$ ) when applying the radiometric calibration to different sections of the spectrum. For lines observed in second order, the right vertical axis is applicable. Note, that the sensitivity ratio between both orders, which is wavelength dependent, has been chosen for (and is only correct for) the central wavelength. Also note, that second order lines are always superimposed on a first order background. We also display in green the BN/CI ratio, which is a useful parameter for electron temperature classification. New identifications for the lines at 1286.20 and $1293.14 \AA$ as Ca V/2 and for lines 1398.06 and $1423.86 \AA$ as S IV have been established during the production process.

c Available via anonymous ftp to cdsarc.u-strasbg.fr (130.79.128.5) http://cdsweb.u-strasbg.fr/cgi-bin/qcat?J/ $\mathrm{A}+\mathrm{A} / 375 / 591$ 

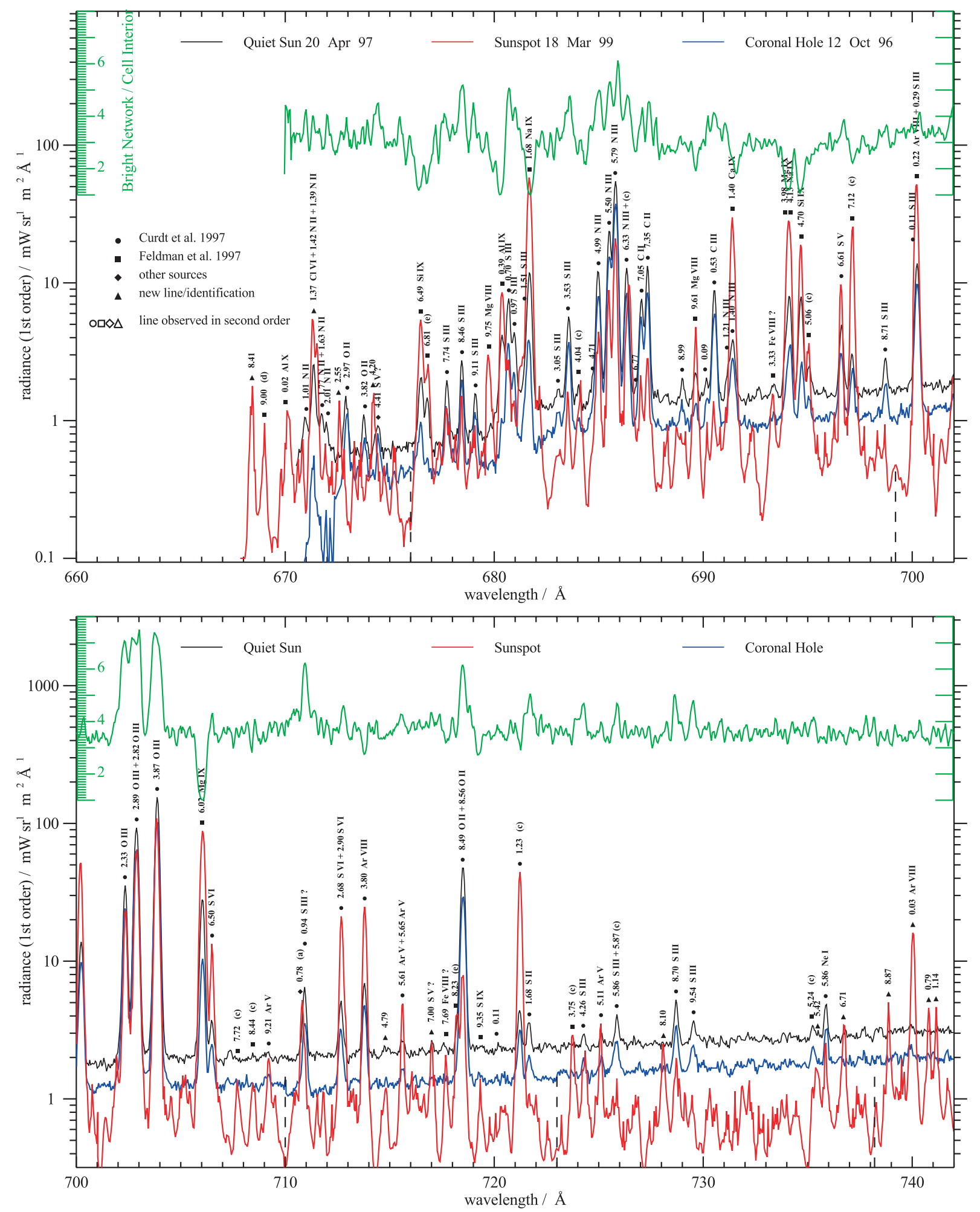

Fig. 4. 

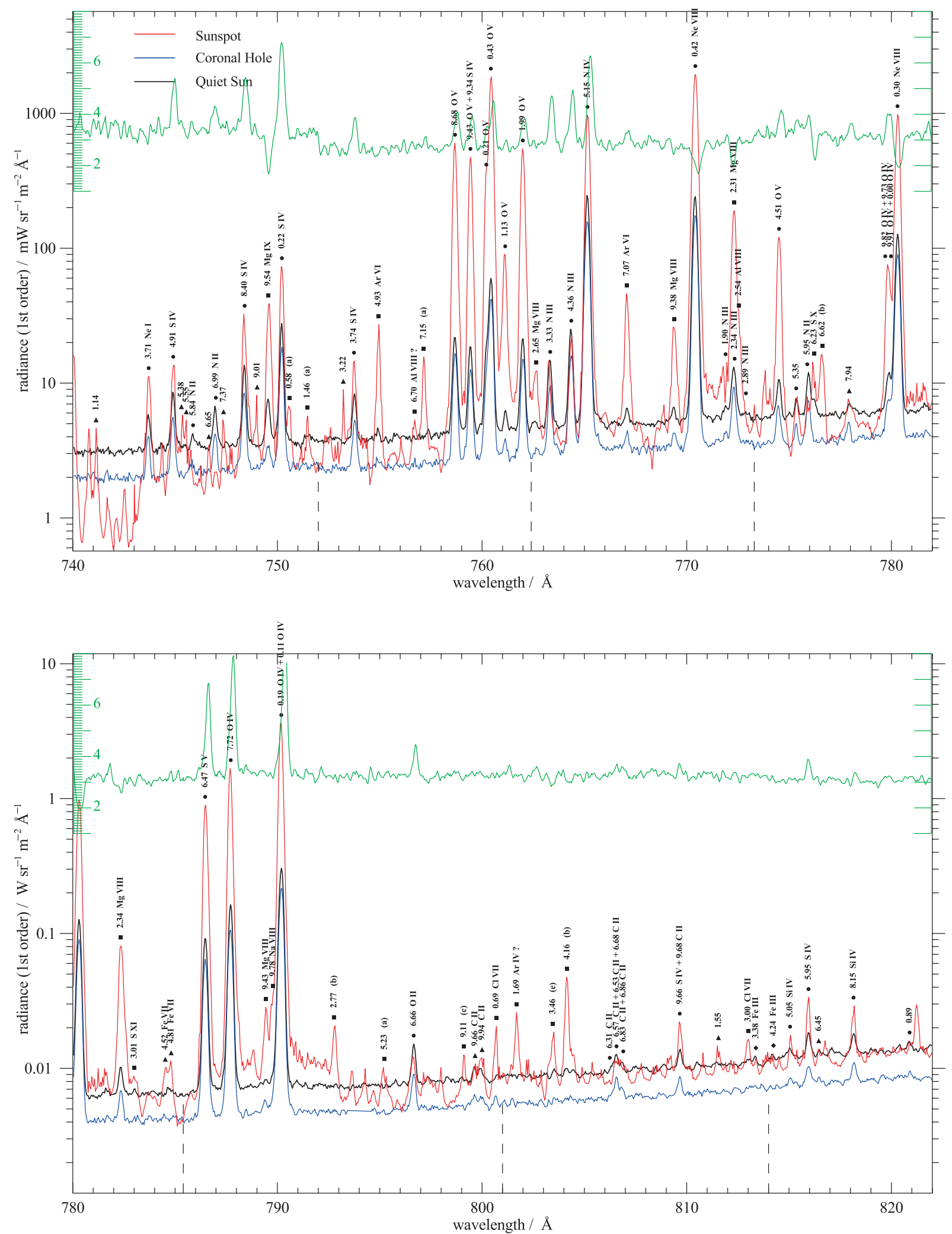

Fig. 4. continued. 
W. Curdt et al.: EUV spectral atlas of the quiet Sun
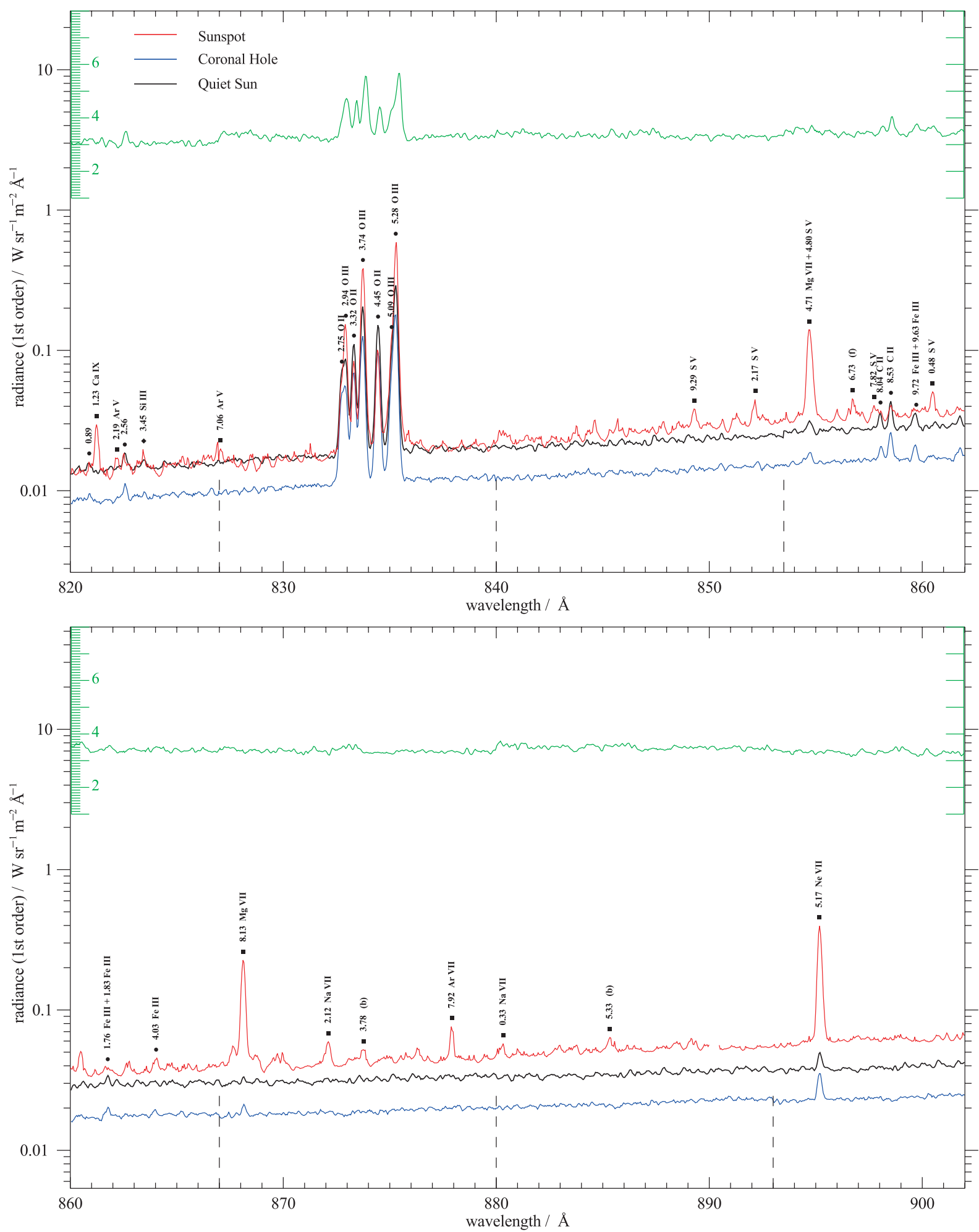

Fig. 4. continued. 

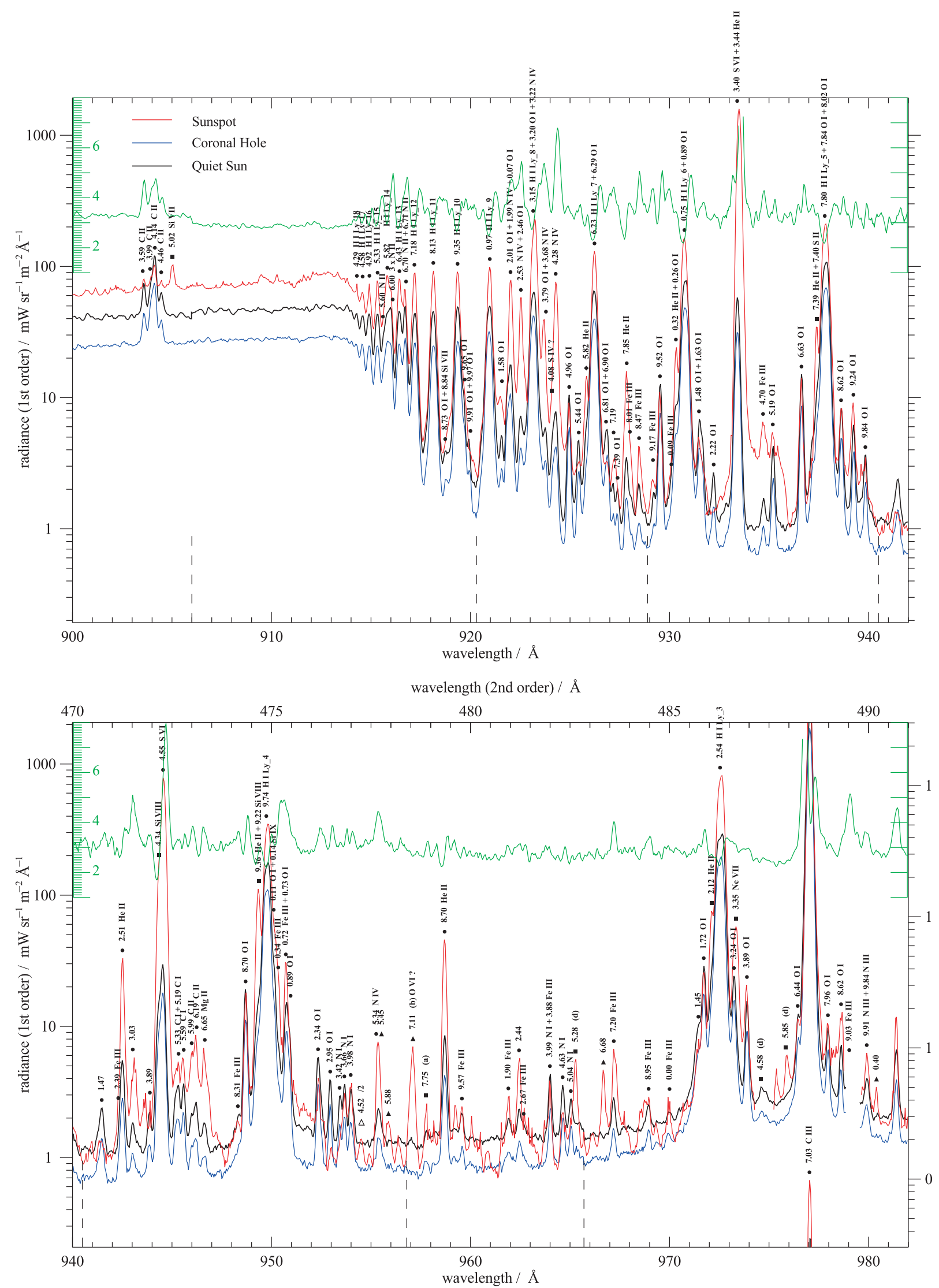

Fig. 4. continued. 

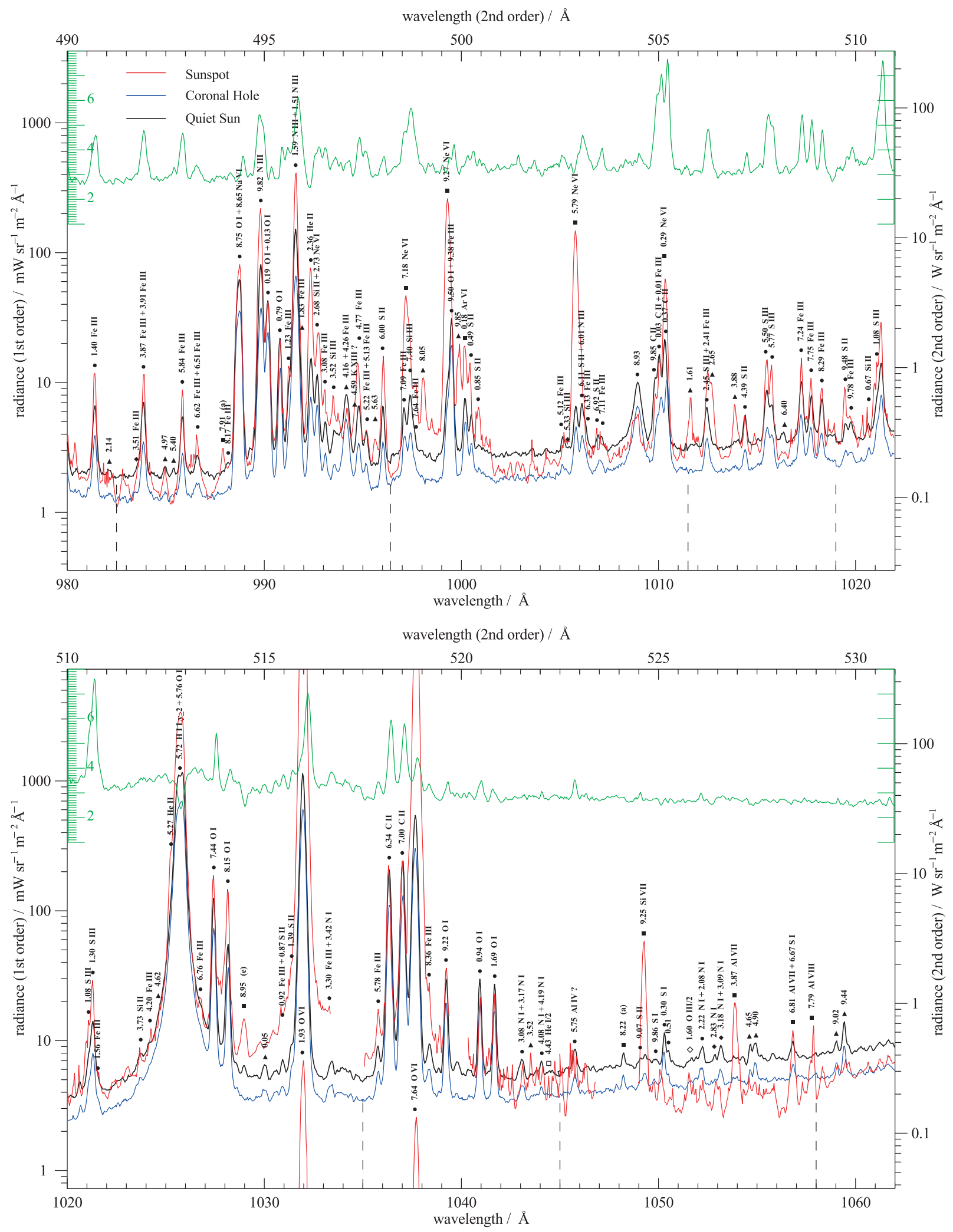

Fig. 4. continued. 

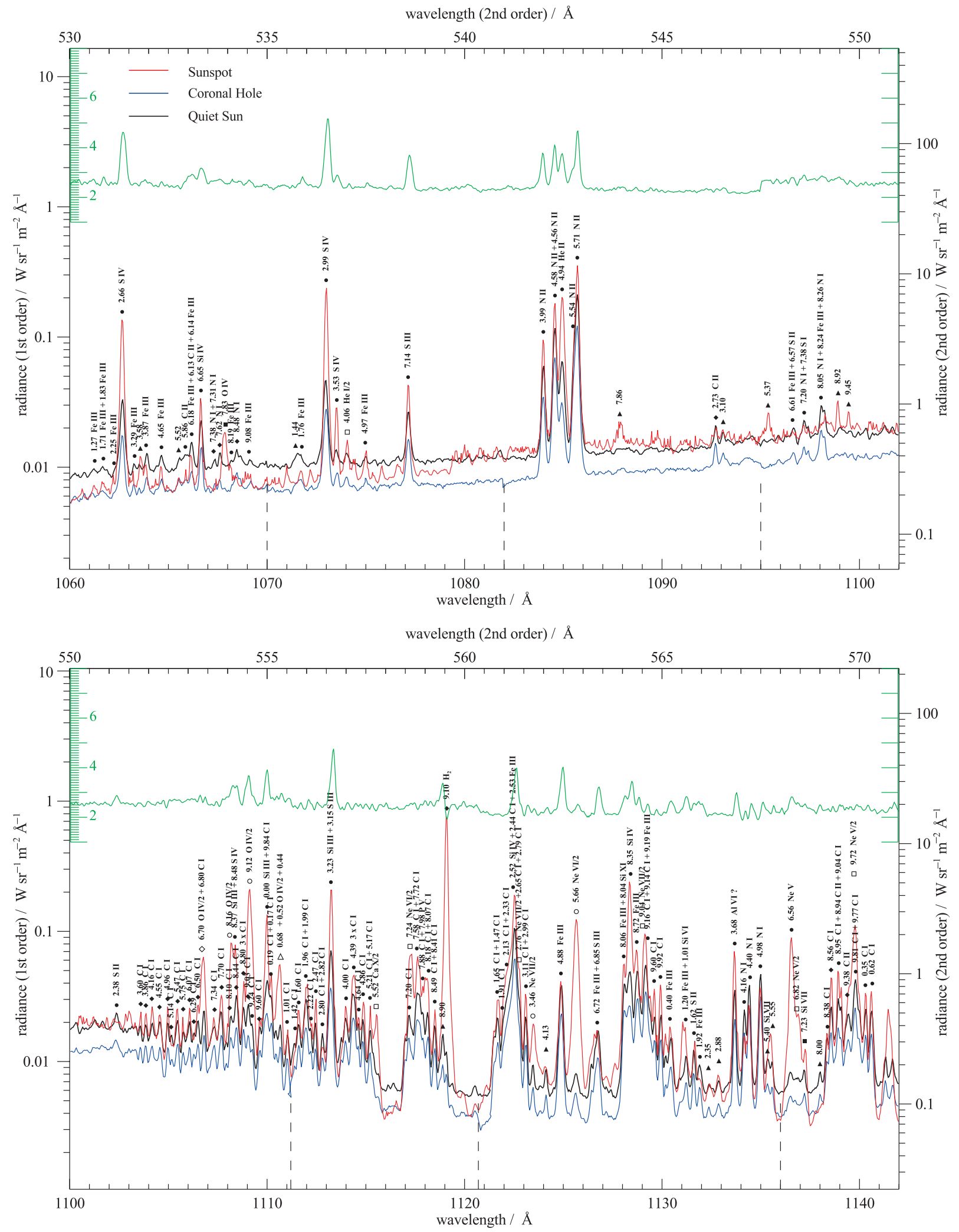

Fig. 4. continued. 

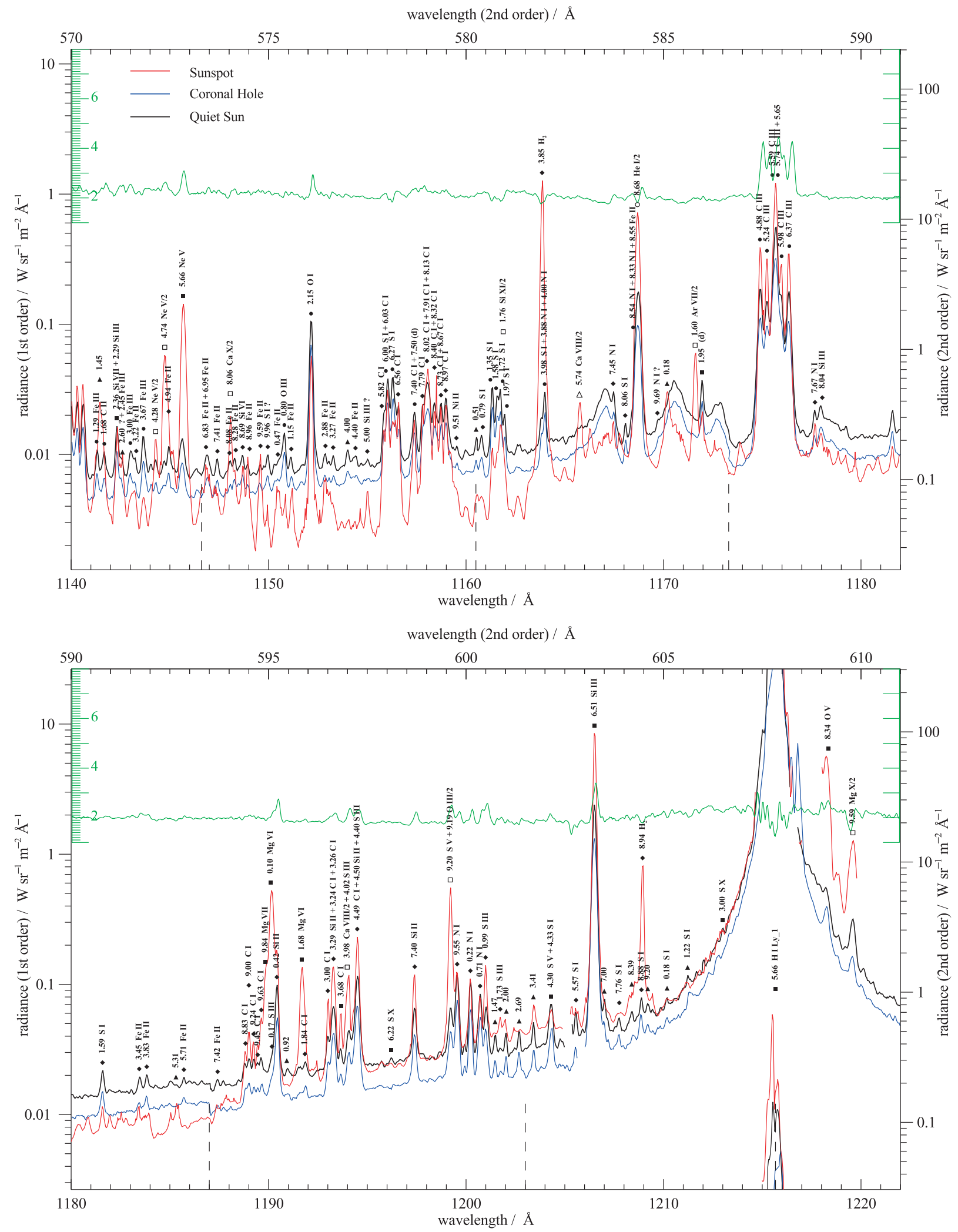

Fig. 4. continued. 

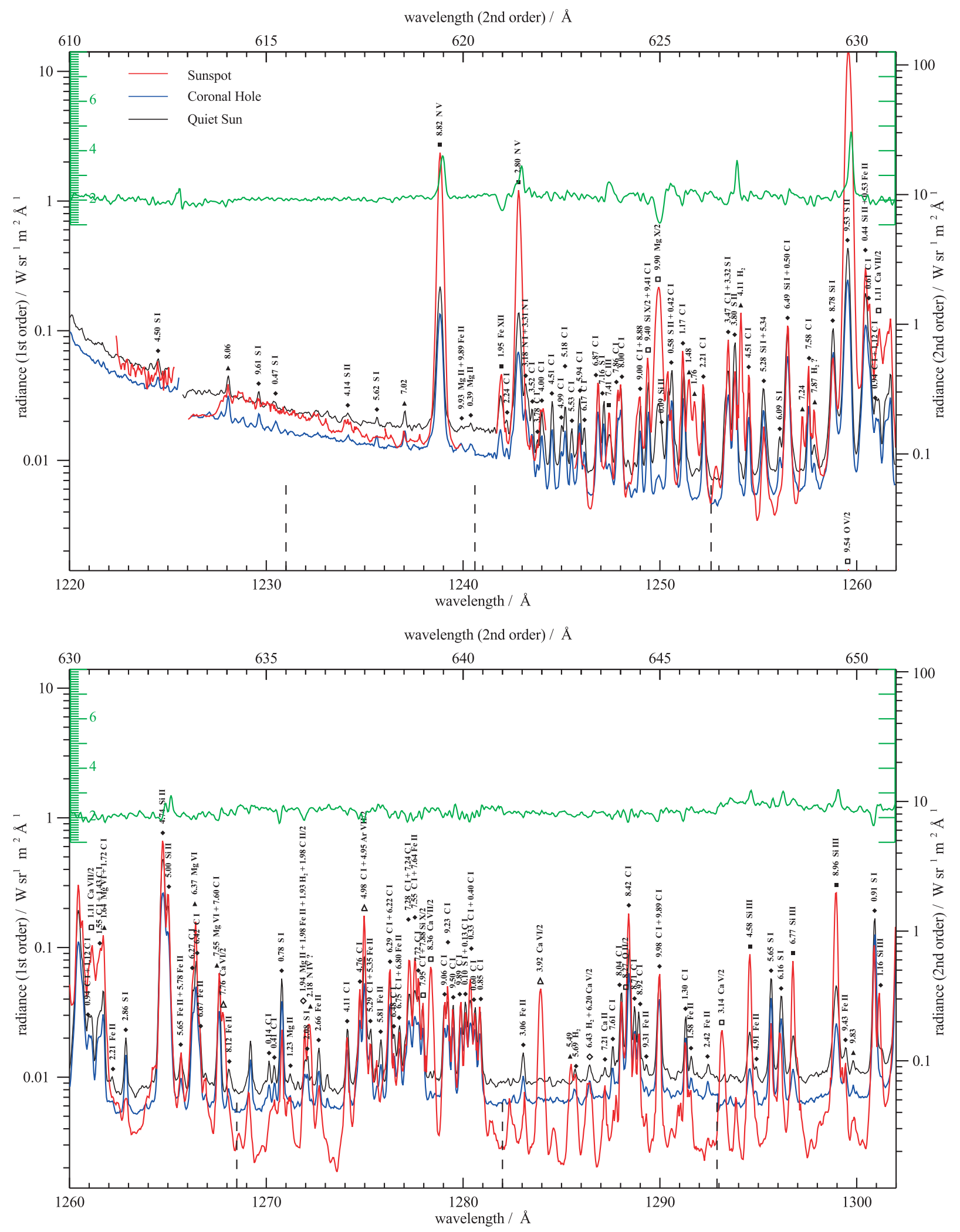

Fig. 4. continued. 

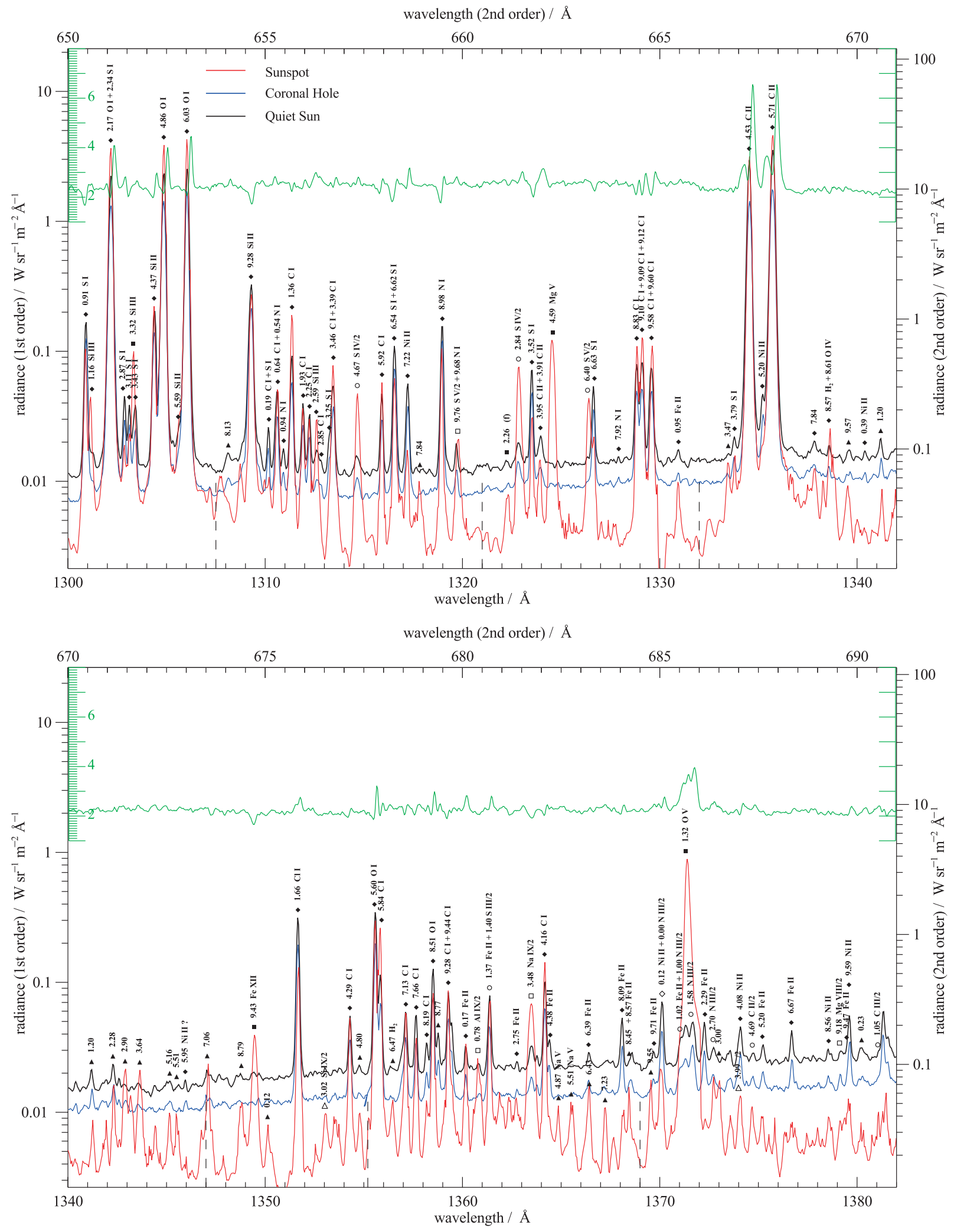

Fig. 4. continued. 

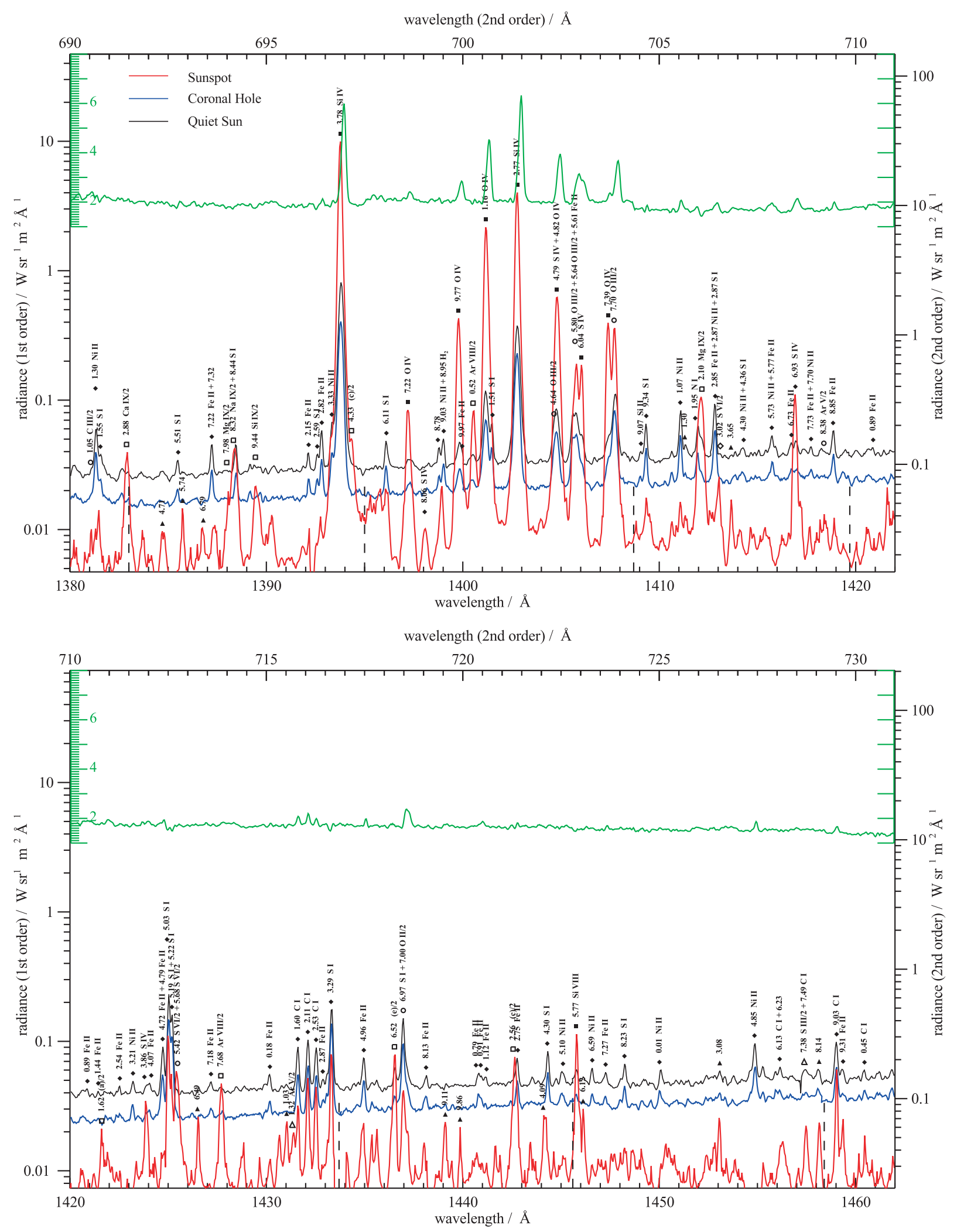

Fig. 4. continued. 

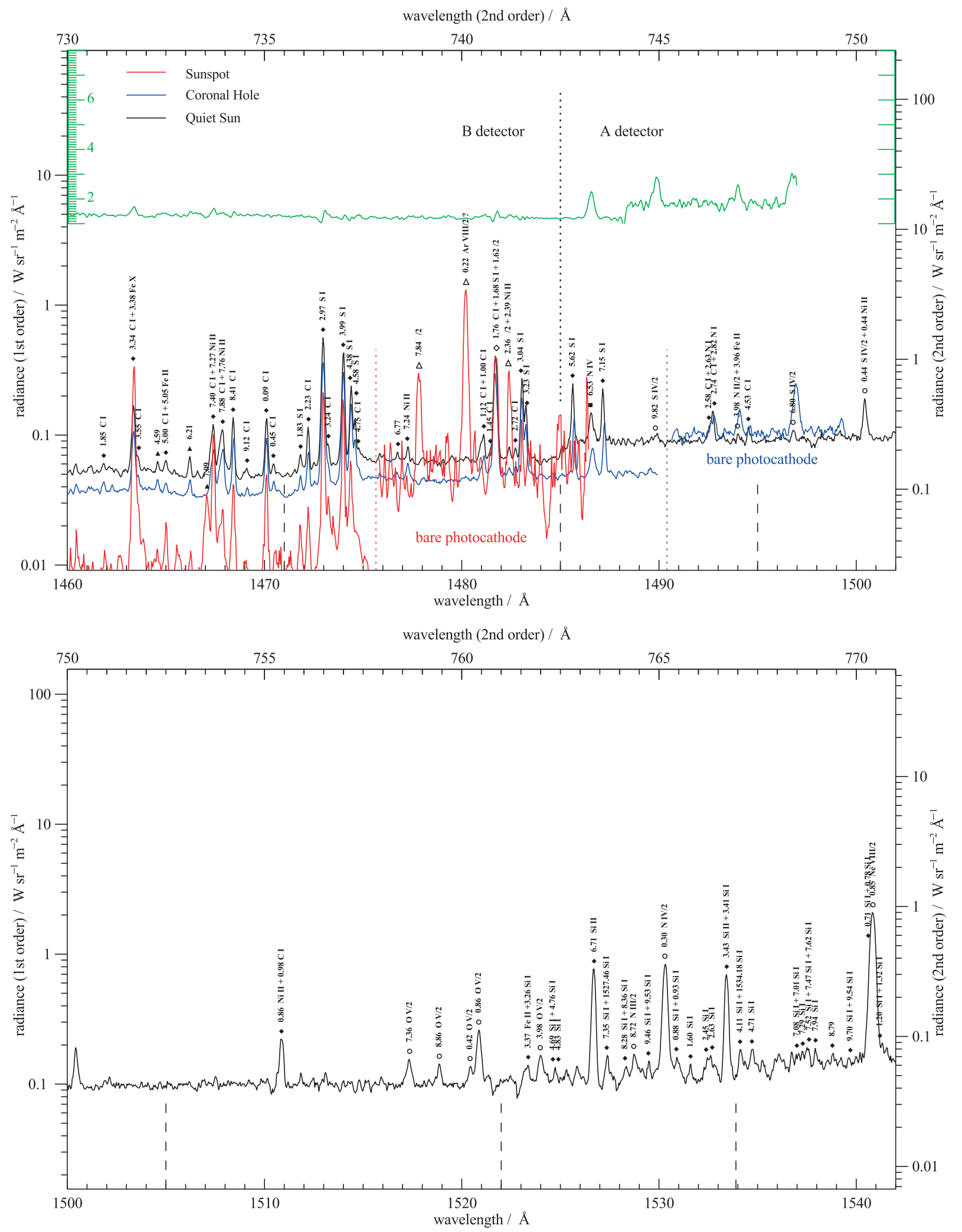

Fig. 4. continued. 

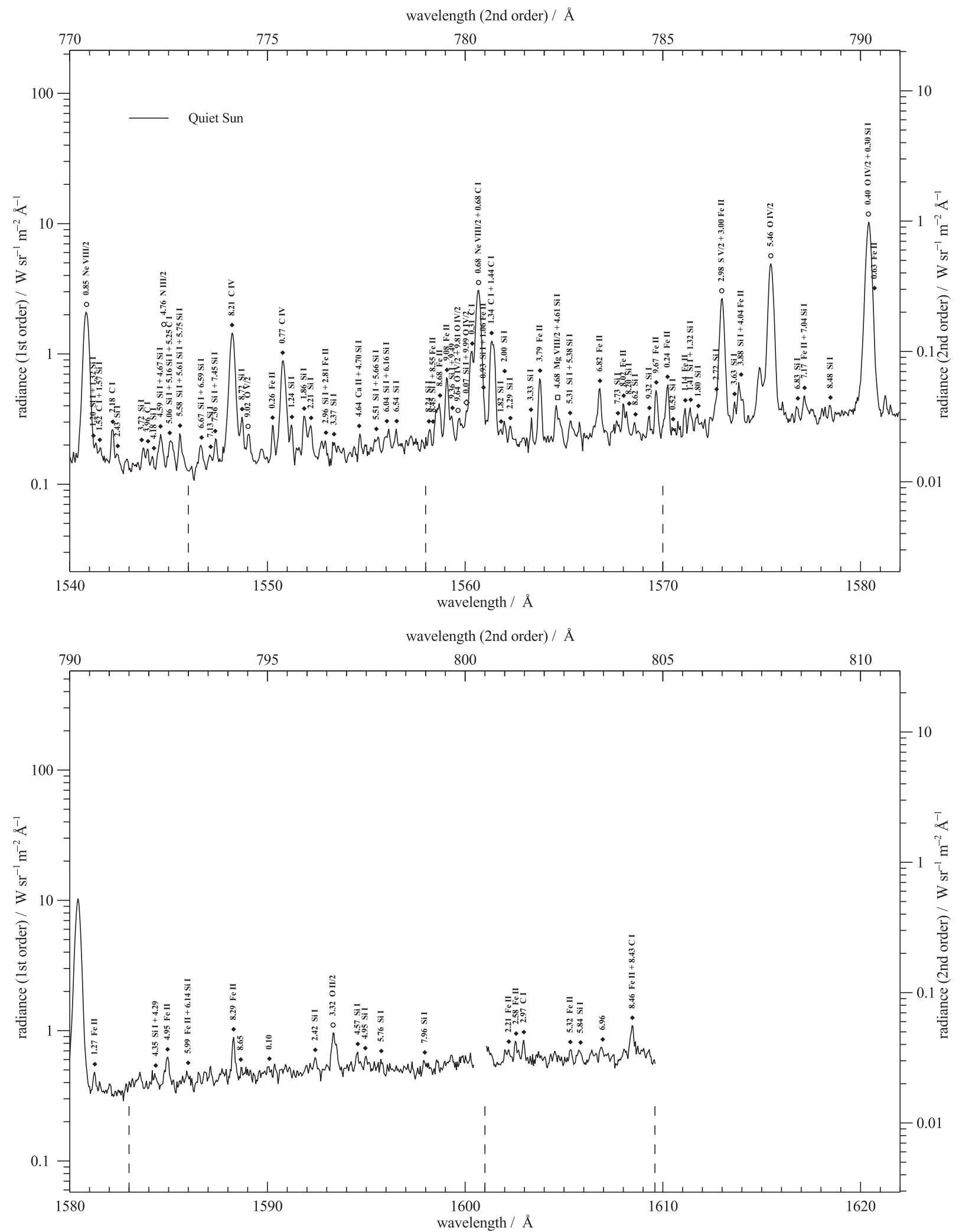

Fig. 4. continued. 


\section{References}

Ayres, T. R. 2000, Solar Phys., 193, 273

Bartoe, J.-D. F., \& Brueckner, G. E. 1975, J. Opt. Soc. Am., $65,13$.

Bartoe, J.-D. F., Brueckner, G. E., Purcell, J. D., \& Tousey, R. 1977, Appl. Opt., 16, 879

Bartoe, J.-D. F., Brueckner, G. E., Nicolas, K. R., et al. 1979, MNRAS, 187, 463

Brekke, P., Kjeldseth-Moe, O., Bartoe, J.-D. F., \& Brueckner, G. E. 1991, ApJS, 75, 1337

Brekke, P. 1993, ApJS, 87, 443

Brekke, P., Thompson, W. T., Woods, T. N., \& Eparvier, F. G. 2000, ApJ, 536, 959

Brooks, D. H., Fischbacher, G. A., Fludra, A., et al. 1999, A\&A, 347, 277

Brueckner, G. E. 1981, in Solar Active Regions, ed. F. Q. Orrall (Colorado Associated University Press, Boulder), 113

Burton, W. M., Ridgeley, A., \& Wilson, R. 1967, MNRAS, 135, 207

Burton, W. M., \& Ridgeley, A. 1970, Solar Phys., 14, 3

Cohen, L., Feldman, U., \& Doschek, G. A. 1978, ApJS, 37, 393

Cohen, L. 1981, An Atlas of Solar Spectra between 1175 and 1950 Angstroms Recorded on Skylab with the NRL's Apollo Telescope Mount Experiment (NASA Ref. Pub.) 1069

Cook, J. W., \& Brueckner, G. E. 1979, ApJ, 227, 645

Curdt, W., Feldman, U., Laming, J. M., et al. 1997, A\&AS, 126,281

Curdt, W., \& Heinzel, P. 1998, ApJ, 503, L95

Curdt, W., Dwivedi, B. N., \& Feldman, U. 2000a, J. Astrophys. Astr., 21 (3\&4), 397

Curdt, W., Landi, E., Wilhelm, K., \& Feldman, U. 2000b, Phys. Rev. A, 62, 022502

Dammasch, I. E., Wilhelm, K., Curdt, W., \& Schühle, U. 1999a, in Magnetic Fields and Solar Processes, Proc. 9th EPS meeting, Florence, 1999, ed. A. Wilson, ESA-SP-448, 1165

Dammasch, I. E., Wilhelm, K., Curdt, W., \& Hassler, D. M. 1999b, A\&A, 346, 285

Delaboudinière, J.-P., Artzner, G. E., Brunaud, J., et al. 1995, Solar Phys., 162, 291

Dere, K. P. 1978, ApJ, 221, 1062

Doschek, G. A., Feldman, U., VanHoosier, M. E., \& Bartoe, J.-D. F. 1976, ApJS, 31, 417

Dupree, A. K., \& Reeves, E. M. 1971, ApJ, 165, 599

Feldman, U., Doschek, G. A., VanHoosier, M. E., \& Purcell, J. D. 1976a, ApJS, 31, 445

Feldman, U., Brown, C. M., Doschek, G. A., et al. 1976b, J. Opt. Soc. Am., 66, 853

Feldman, U., Doschek, G. A. 1978, ApJS, 37, 443

Feldman, U., Purcell, J. D., \& Dohne, B. 1987, An Atlas of Extreme Ultraviolet Spectroheliograms from 170-625 ̊, NRL Report

Feldman, U., Doschek, G. A., \& Seely, J. S. 1988, J. Opt. Soc. Am., 5, 2237

Feldman, U., \& Doschek, G. A. 1991, ApJS, 75, 925

Feldman, U., Behring, W. E., Curdt, W., et al. 1997, ApJS, 113,195
Feldman, U., Doschek, G. A., Schühle, U., \& Wilhelm, K. 1999, ApJ, 518, 500

Feldman, U., Curdt, W., Landi, E., \& Wilhelm, K. 2000, ApJ, 544,508

Gabriel, A. H., Garton, W. R. S., Goldberg, L., et al. 1971, ApJ, 169, 595

Harrison, R. A., et al. 1995, Solar Phys., 162, 233

Hassler, D. M., Rottman, G. J., \& Orrall, F. Q. 1991, ApJ, 372,710

Hollandt, J., Schühle, U., Paustian, W., et al. 1996, Appl. Opt., 35,5125

Huber, M. C. E., Dupree, A. K., Goldberg, L., et al. 1973, ApJ, 183,291

Innes, D. E., Inhester, B., Axford, W. I., \& Wilhelm, K. 1997, Nature, 386, 811

Kelly, R. L. 1987, J. Phys. Chem. Ref. Data, 16, 1

Kjeldseth-Moe, O., VanHoosier, M. E., Bartoe, J.-D. F., \& Brueckner, G. E. 1976, A Spectral Atlas of the Sun between 1175-2100 ̊, NRL Report 8056

Lemaire, P., Wilhelm, K., Curdt, W., et al. 1997, Solar Phys., 170,105

Malinovsky, M., \& Heroux, L. 1973, ApJ, 181, 1009

Mariska, J. T. 1992, The Solar Transition Region (Cambridge University Press, Cambridge)

Moran, T. 1996, private communication

Peter, H., \& Judge, P. G. 1999, ApJ, 522, 1148

Reeves, E. M., Huber, M. C. E., \& Timothy, J. G. 1977, Appl. Opt., 16, 837

Samain, D. 1991, A\&A, 244, 217

Sandlin, G. D., Bartoe, J.-D. F., Brueckner, G. E., et al. 1986, ApJS, 61, 801

Schühle, U., Brekke, P., Curdt, W., et al. 1998, Appl. Opt., 37, 2646

Schühle, U., Curdt, W., Wilhelm, K., et al. 1999, Space Sci. Rev., 87, 299

Schühle, U., Curdt, W., Hollandt, J., et al. 2000, Appl. Opt., 39,418

Siegmund, O. H. W., Gummin, M. A., Stock, J. M., et al. 1994, Proc. SPIE 2280, 89

Thomas, R. J., \& Neupert, W. 1994, ApJS, 91, 461

Tousey, R., Bartoe, J.-D.F., Brueckner, G. E., \& Purcell, J. D. 1977, Appl. Opt., 16, 870

Vernazza, J. E., \& Reeves, E. M. 1978, ApJS, 37, 485

Wilhelm, K., Curdt, W., Marsch, E., et al. 1995, Solar Phys., 162, 189

Wilhelm, K., Lemaire, P., Feldman, U., et al. 1997a, Appl. Opt., 36, 6416

Wilhelm, K., Lemaire, P., Curdt, W., et al. 1997b, Solar Phys., 170, 75

Wilhelm, K., Lemaire, P., Dammasch, I. E., et al. 1998, A\&A, 334,685

Wilhelm, K., Woods, T. N., Schühle, U., et al. 1999, A\&A, 352, 321

Wilhelm, K., Schühle, U., Curdt, W., et al. 2000, Metrologica, $37(5), 393$

Woods, T. N., Rottman, G. J., Bailey, S. M., et al. 1998a, Solar Phys., 177, 133

Woods, T. N. 1998b, private communication 